\title{
Reinstatement of the New Zealand cave wētā genus Miotopus Hutton (Orthoptera: Rhaphidophoridae) and description of a new species
}

\author{
Josephine L. FITNESS ${ }^{1}$, Mary MORGAN-RICHARDS ${ }^{2}$, \\ Danilo HEGG $^{3}$ \& Steven A. TREWICK ${ }^{4, *}$ \\ ${ }^{1,2,4}$ Ecology Group, Massey University, Private Bag 11-222, Palmerston North 4442, New Zealand. \\ ${ }^{3} 135$ Blacks Road, Dunedin 9010, New Zealand. \\ *Corresponding author: s.trewick@massey.ac.nz \\ ${ }^{1}$ Email: josephine.fitness@gmail.com \\ 22Email: M.Morgan-Richards@massey.ac.nz \\ 33Email: danilo_hegg@hotmail.com \\ ${ }^{1}$ urn:lsid:zoobank.org:author:8668015A-BAE6-4673-AF99-42EA57FB581D \\ ${ }^{2}$ urn:1sid:zoobank.org:author:48F2FB1A-4C03-477C-8564-5417F9739AE1 \\ ${ }^{3}$ urn:lsid:zoobank.org:author:34DFC18A-F53D-417F-85FC-EF514F6D2EFD \\ ${ }^{4}$ urn:1sid:zoobank.org:author:7A378EE1-BADB-459D-9BAA-7059A675F683
}

\begin{abstract}
Comparison of morphological and genetic data from New Zealand forest cave wêtā suggests we should recognise the genus Miotopus proposed by Hutton (1898). A new species within this genus is described (Miotopus richardsi sp. nov.). Both Miotopus diversus (Hutton, 1898) and Miotopus richardsi sp. nov. are common in native forests and widespread in New Zealand. Here we provide their known distributions and key traits.
\end{abstract}

Keywords. Cave wētā, cave cricket, Miotopus, Pleioplectron, Rhaphidophoridae.

Fitness J.L., Morgan-Richards M., Hegg D. \& Trewick S.A. 2018. Reinstatement of the New Zealand cave wētā genus Miotopus Hutton (Orthoptera: Rhaphidophoridae) and description of a new species. European Journal of Taxonomy 468: 1-24. https://doi.org/10.5852/ejt.2018.468

\section{Introduction}

Identifying cave wētā to genus level is made difficult by a limited number of accessible morphological and diagnostically informative characters and very brief descriptions for many of the nineteen genera of New Zealand Rhaphidophoridae (Cook et al. 2010). Hutton (1897) described the New Zealand rhaphidophorid genus Pleioplectron including four species. A year later, he transferred one of these, Pleioplectron diversum to the new genus Miotopus as Miotopus diversus (Hutton 1898). Hutton cited the presence of the fore femoral retrolateral spine and the subapical inferior pair of spines on the hind tibia as evidence of sufficient differentiation. However, Richards (1959) disagreed and returned Miotopus diversus to Pleioplectron stating that the differences in spination identified by Hutton (1898) to establish Miotopus represented congeneric variation. 
In his generic synopsis and species description Hutton (1897) made inconsistent reference to apical spines (see Fig. 1). He described Pleioplectron as having one apical spine on the fore femora, two on the middle femora and none on hind femora, and two pairs on each of the fore and middle tibiae, three pairs on the hind tibiae. These are not mentioned in the species descriptions except that $P$. diversum (= Miotopus diversus) has a pair of short apical spines on the fore femora. Richards (1959) noted that Pleioplectron, including Hutton's type specimens, have a prolateral apical spine beneath the hind femora, but this probably reflects a difference of definition. Spination on the hind femora is quite different from fore and mid femora in all New Zealand species of Rhaphidophoridae; the hind legs being specialised for jumping. Hutton (1897) stated that Pleioplectron's hind tibiae have three pairs of apical spines but Richards (1959) gave four pairs. Again, this most likely reflects a difference in definition, because some taxonomists interpret the pair of superior subapical spines (S21 \& S22 in Fig. 1) as the first superior linear spines (see Fig. 2). Hutton generally presented very simple outline drawings of structures, whereas Richards used very detailed drawings of only the hind portion of the abdomen. Modern taxonomy benefits from high quality photographic imagery that helps precisely indicate what structure is being referred to and provides information of colour, patterning and texture.

We have used morphological, spatial and genetic data to review the status of New Zealand Rhaphidophoridae, and found support for Miotopus Hutton, 1898 and an additional species in this genus common on South Island which we describe here.

\section{Material and methods}

Cave wētā were collected opportunistically around New Zealand using day and night searching of forests and caves, and pitfall trapping. More than 3500 specimens have been catalogued, of which about 1000 have been examined in detail including $>154$ putative Pleioplectron. Cave wêtā material is held in the Phoenix Lab collection at Massey University, with the exception of type material lodged at Te Papa Tongarewa Museum of New Zealand (NMNZ AI.035053 and AI.035054). Relevant specimens were identified based on descriptions by Hutton $(1897,1898)$ and Richards (1959), and were examined under a dissecting microscope so that adult males and females could be identified. Distinguishing between adults and large juveniles relied on the darker sclerotised bodies and fully formed external genital structures of the former. In particular the pigmentation, shape and sharpness of ovipositors, subgenital plates and cerci were informative about the developmental stage. We looked for the presence/absence of each of 22 apical leg spines (Fitness et al. 2015) (Fig. 1), and the combinations and numbers of linear spines on legs, characteristics of antennae and shape of subgenital plate and suranal plate.

\section{Collection acronyms}

iNaturalist $=$ Available from iNaturalist.org [accessed 1 Sep. 2018]

LCR $=$ Landcare Research, Wellington, New Zealand

MPN $\quad=$ Phoenix Lab, Massey University, Palmerston North, New Zealand

NMNZ $\quad=$ Te Papa Tongarewa Museum, Wellington, New Zealand

We also extracted genomic DNA from representative morphotypes using a salting-out protocol (Trewick \& Morgan-Richards 2005). For each sample, a fragment upto $\sim 1500$ base pairs (bp) of the mitochondrial cytochrome $c$ oxidase I (COI) gene of the mitochondrial genome was amplified using polymerase chain reaction (PCR) with combinations of universal invertebrate primers: LCO1490 (Folmer et al. 1994), C1-N-2191, C1-J-2195 and L2-N-3014 (Simon et al. 1994). Successful PCR products were prepared using the SAP/EXO1 digest protocol (USB Corp., Cleveland, OH) and sequenced with Bigdye chemistry and an ABI 3730 genetic analyser (Applied Biosystems Inc., Carlsbad, CA). Nucleotide sequences were assembled and aligned using Geneious 'ver.' 9 (Kearse 


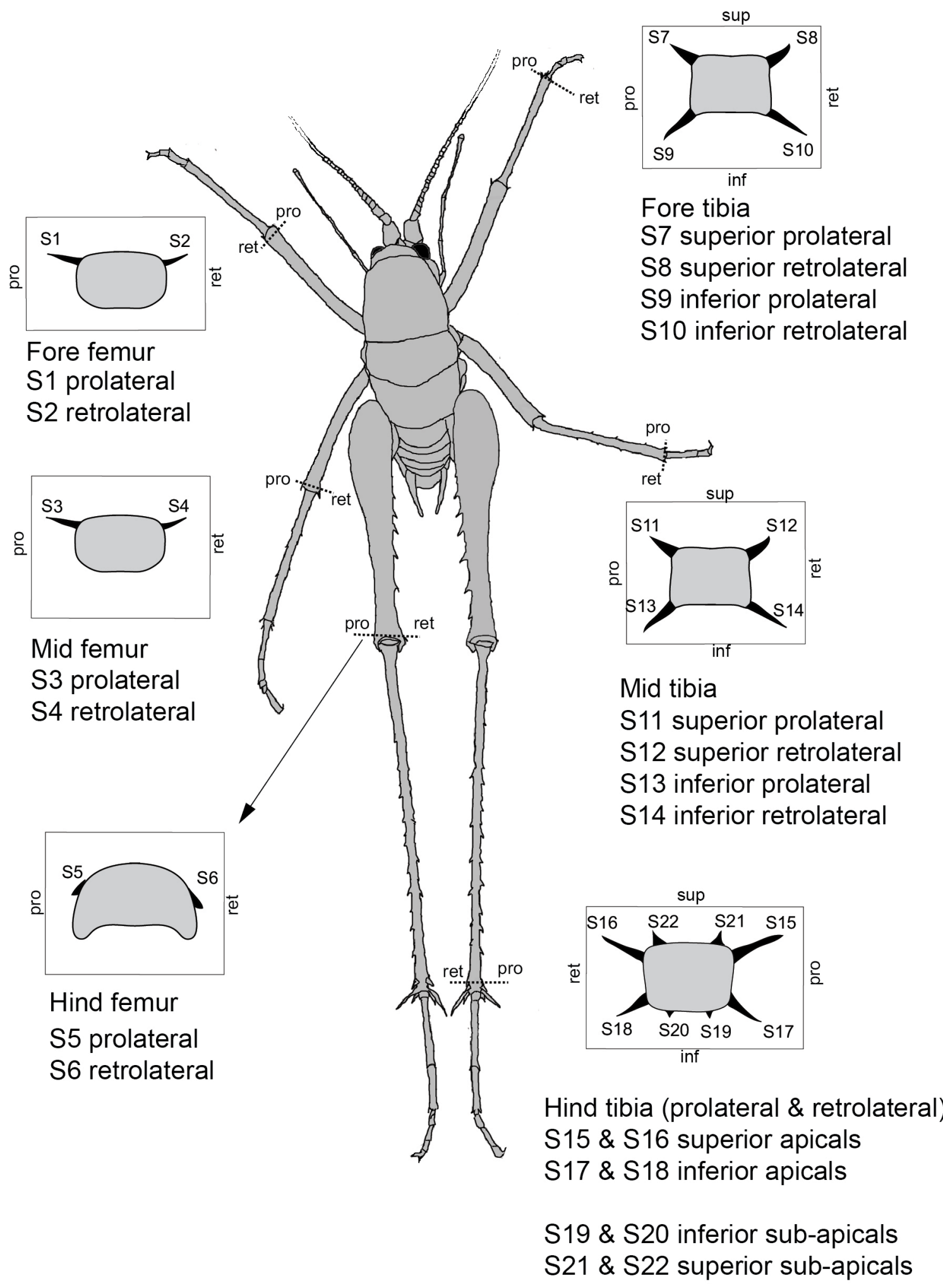

Fig. 1. Apical spine numbering, terminology and position on Rhaphidophoridae, after Fitness et al. (2015). Dorsal view, showing cross-sectional relationship of each potential spine on femora and tibiae. Positions are indicated as prolateral (anterior facing), retrolateral (posterior facing), inferior (ventral facing) and superior (dorsal facing) orientations are indicated. 
et al. 2012). No insertions/deletions were detected and sequences were translated to confirm that there were no stop codons or frame shifts that would indicate the presence of nuclear paralogs. We examined the relationships of putative Miotopus mtDNA COI haplotypes with respect to Pleioplectron and other representatives of the New Zealand cave wētā (Allegrucci et al. 2010) using phylogenetic reconstruction using Maximum Likelihood and Bayesian inference algorithms implemented in Geneious 'ver.' 9.

\section{Results}

Using combinations of apical leg spines and general appearance (Table 1) we identified cave wêtā that could be assigned to Miotopus diversus, Pleioplectron hudsoni Hutton, 1896 and P. simplex Hutton, 1896. Although specimens of Miotopus diversus are broadly similar in appearance to Pleioplectron, including the comparatively short and stout antennae of males, there are clear differences. Miotopus diversus differs from Pleioplectron by a distinctive apical spine count (Miotopus has 2 apical spines on the fore femur, whereas Pleioplectron has 1), the presence of dorsal spines on the mid tibia, the subgenital plate shape and the larger size of adults. Females of Miotopus and Pleioplectron have a different number and shape of serrations towards the ventral tip of the ovipositor. Specimens found at

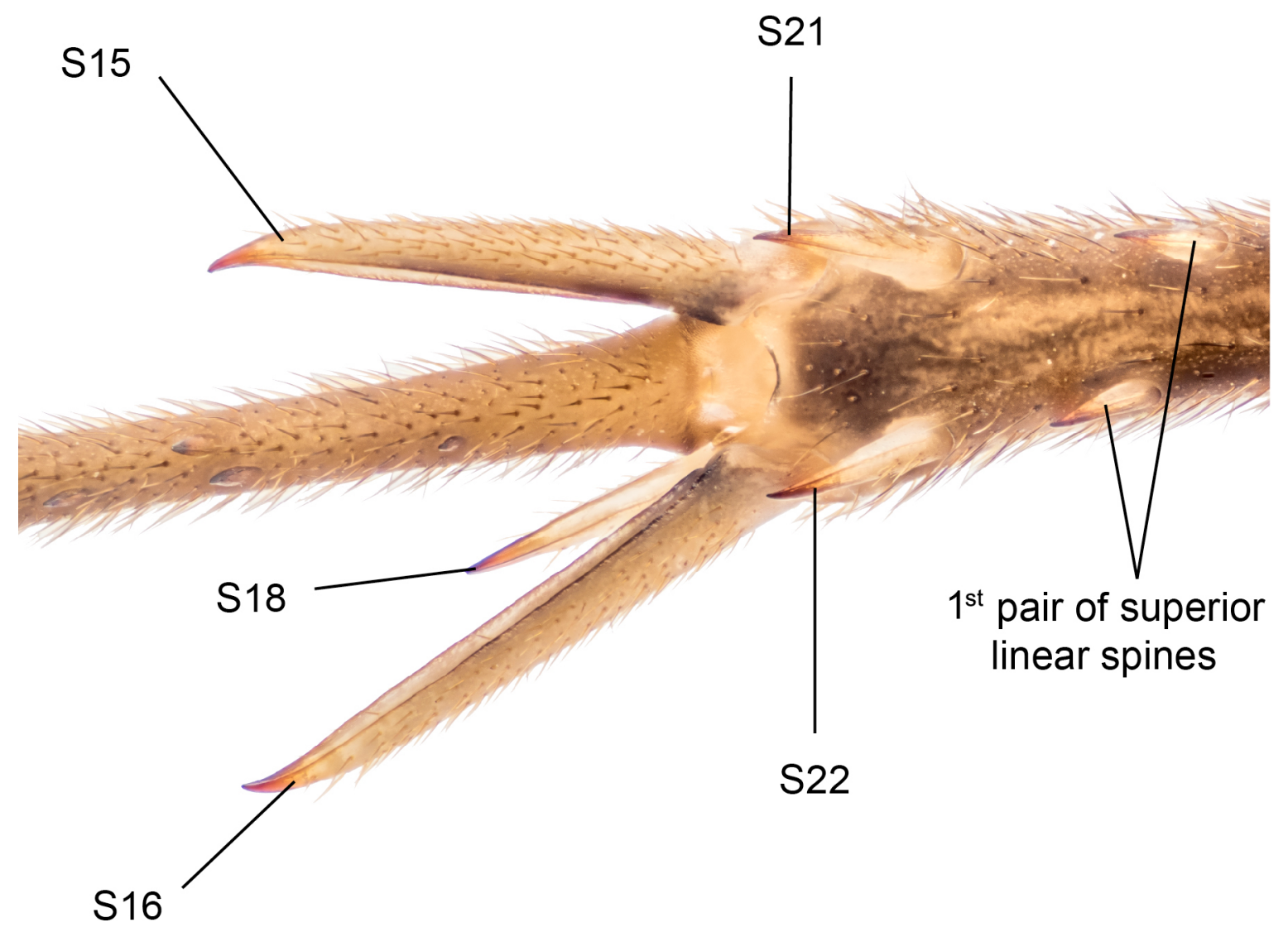

Fig. 2. Apical spines on the left hind tibia of Pleioplectron simplex Hutton, 1896 (MPN CW3459), numbered as in Fig. 1 (from Fitness et al. 2015). Dorsal view of posterior distal section of left hind tibia including part of first tarsal segment. Four pairs of 'apical' spines are commonly present: the inferior subapical pair S19 \& S20 (not visible here); inferior apical pair S17 \& S18 (S17 obscured here); superior apical pair S15 \& S16; superior subapical pair S21 \& S22. Some taxonomists have treated S21 \& S22 as the first pair of superior linear spines, resulting in recording of three pairs of 'apical' rather than four. 
Table 1. Dimensions and spine counts of Pleioplectron Hutton, 1896 and Miotopus Hutton, 1898 Rhaphidophoridae. *(4-4-7 in two Lewis Pass specimens).

\begin{tabular}{|c|c|c|c|c|c|}
\hline & & $\begin{array}{l}\text { Pleioplectron } \\
\text { simplex }\end{array}$ & $\begin{array}{c}\text { Pleiopletron } \\
\text { hudsoni }\end{array}$ & $\begin{array}{c}\text { Miotopus } \\
\text { diversus }\end{array}$ & $\begin{array}{c}\text { Miotopus } \\
\text { richardsi sp. nov. }\end{array}$ \\
\hline \multicolumn{2}{|l|}{ Sample size } & $7(3$ 우, $4 \overbrace{}^{\lambda} \overbrace{}^{\lambda})$ & $11(5$ 우, 6 ठึ่ $)$ & 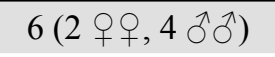 & $12\left(7\right.$ 우, $\left.5 \bigcirc^{\Uparrow}\right)$ \\
\hline \multicolumn{2}{|c|}{ Apical spines fore and mid femur } & 1011 & 1011 & 1111 & 1111 \\
\hline \multicolumn{2}{|c|}{ Apical spines tibiae } & $4-4-8$ & $4-4-6$ & $4-4-8$ & $4-4-8^{*}$ \\
\hline \multicolumn{2}{|l|}{ Body length (mm) } & $14.5(13.7-15.0)$ & $9.4(8.4-11.1)$ & $13.55(11.1-14.5)$ & $15.5(14.0-16.5)$ \\
\hline \multicolumn{2}{|l|}{ Pronotum length (mm) } & $4.5(3.8-5.3)$ & $3.3(2.8-3.6)$ & $4.85(3.9-5.9)$ & $5.0(4.5-5.9)$ \\
\hline \multicolumn{2}{|l|}{ Ovipositor length (mm) } & $10.2(9.9-10.7)$ & $7.4(6.8-8.1)$ & $11.55(11.3-11.8)$ & $15.75(13.9-17.0)$ \\
\hline \multicolumn{2}{|c|}{ Ratio ovipositor to body length } & $0.71(0.70-0.71)$ & $0.78(0.65-0.79)$ & $0.80(0.78-0.81)$ & $1.02(0.96-1.10)$ \\
\hline \multicolumn{2}{|c|}{ Teeth: ventral valve of ovipositor } & $5(5-6)$ & $8(7-9)$ & $7.5(7-8)$ & $24(18-30)$ \\
\hline \multicolumn{2}{|c|}{ Teeth: dorsal valve of ovipositor } & 0 & 0 & 0 & 0 \\
\hline \multicolumn{2}{|c|}{ Length of hind tibia (mm) } & $14.3(12.4-16.4)$ & $11.2(10.1-12.1)$ & $15.65(13.0-17.4)$ & $23.35(19.9-29.2)$ \\
\hline \multicolumn{2}{|c|}{ Ratio hind tibia to body length } & $0.99(0.84-1.13)$ & $1.18(1.05-1.35)$ & $1.2(1.0-1.3)$ & $1.5(1.3-1.8)$ \\
\hline $\begin{array}{l}\text { Superior spines } \\
\text { hind tibia }\end{array}$ & $\begin{array}{l}\text { prolateral } \\
\text { retrolateral }\end{array}$ & $\begin{array}{l}24(20-29) \\
27(23-32)\end{array}$ & $\begin{array}{l}20(18-25) \\
21(19-24)\end{array}$ & $\begin{array}{l}31(29-33) \\
33(28-34)\end{array}$ & $\begin{array}{l}36(31-41) \\
38(35-44)\end{array}$ \\
\hline $\begin{array}{l}\text { Spine density on hind } \\
\text { tibia (count/ } \mathrm{mm} \text { ) }\end{array}$ & $\begin{array}{l}\text { prolateral } \\
\text { retrolateral }\end{array}$ & $\begin{array}{l}1.6(1.5-1.9) \\
2.0(1.5-2.1)\end{array}$ & $\begin{array}{l}1.79(1.59-2.38) \\
1.96(1.65-2.11)\end{array}$ & $\begin{array}{l}2.0(1.7-2.5) \\
2.2(1.6-2.5)\end{array}$ & $\begin{array}{l}1.5(1.3-1.8) \\
1.7(1.4-1.9)\end{array}$ \\
\hline \multicolumn{2}{|c|}{ Pairs of articulated spines on hind tibia } & 0 & 0 & 0 & $3(3-3)$ \\
\hline \multicolumn{2}{|c|}{ Superior spines on $1^{\text {st }}$ tarsus segment } & $8(5-11)$ & $8(7-11)$ & $8(8-8)$ & $7(4-11)$ \\
\hline \multicolumn{2}{|c|}{ Superior spines on $2^{\text {nd }}$ tarsus segment } & $4(0-5)$ & $3(2-4)$ & $2(2-3)$ & $0(0-1)$ \\
\hline $\begin{array}{l}\text { Fore tibia, } \\
\text { inferior spines }\end{array}$ & $\begin{array}{l}\text { prolateral } \\
\text { retrolateral }\end{array}$ & $\begin{array}{l}3(3-3) \\
3(3-3)\end{array}$ & $\begin{array}{l}1(0-1) \\
2(2-2)\end{array}$ & $\begin{array}{c}2(2-2) \\
3.5(3-4)\end{array}$ & $\begin{array}{l}2(2-3) \\
2(1-3)\end{array}$ \\
\hline \multicolumn{2}{|c|}{ Fore tibia, superior spines } & 0 & 0 & 0 & 0 \\
\hline $\begin{array}{l}\text { Mid tibia, } \\
\text { inferior spines }\end{array}$ & $\begin{array}{l}\text { prolateral } \\
\text { retrolateral }\end{array}$ & $\begin{array}{l}3(3-3) \\
3(2-3)\end{array}$ & $\begin{array}{l}2(1-3) \\
1(0-2)\end{array}$ & $\begin{array}{c}3(3-4) \\
2.5(2-3)\end{array}$ & $\begin{array}{l}2(1-2) \\
2(2-3)\end{array}$ \\
\hline $\begin{array}{l}\text { Mid tibia, } \\
\text { superior spines }\end{array}$ & $\begin{array}{l}\text { prolateral } \\
\text { retrolateral }\end{array}$ & 0 & 0 & $\begin{array}{l}1.5(1-3) \\
5(3-6)\end{array}$ & 0 \\
\hline $\begin{array}{l}\text { Hind femur, } \\
\text { inferior spines }\end{array}$ & $\begin{array}{l}\text { prolateral } \\
\text { retrolateral }\end{array}$ & $\begin{array}{c}0 \\
3(2-4), \text { v. small }\end{array}$ & $\begin{array}{c}0 \\
1(0-1), \text { minute }\end{array}$ & $\begin{array}{c}0 \\
1.5(1-3), \text { small } \\
\end{array}$ & $\begin{array}{c}0 \\
1.5(1-2), \text { small } \\
\end{array}$ \\
\hline
\end{tabular}


several locations on North Island are consistent with Hutton's description of Miotopus diversus, but most specimens from South Island represent an undescribed species.

In the OR our phylogenetic analysis of a $1435 \mathrm{bp}$ alignment of mtDNA COI, Miotopus diversus did not cluster with Pleioplectron hudsoni and P. simplex (Fig. 3). Instead, other New Zealand Rhaphidophoridae (Talitropsis Bolívar, 1882 and Pachyrhamma Brunner von Wattenwyl, 1888) included in the analysis fell between the P. hudsoni / P. simplex clade and the Miotopus lineage. A second putative Miotopus taxon grouped with M. diversus although substitution saturation at the COI locus results in posterior probabilities at nodes $<1$. We also examined clustering of COI haplotypes $(835 \mathrm{bp}$ ) from 41 putative Miotopus sampled from around New Zealand and this revealed one cluster consisted of all specimens identified as M. diversus, including some

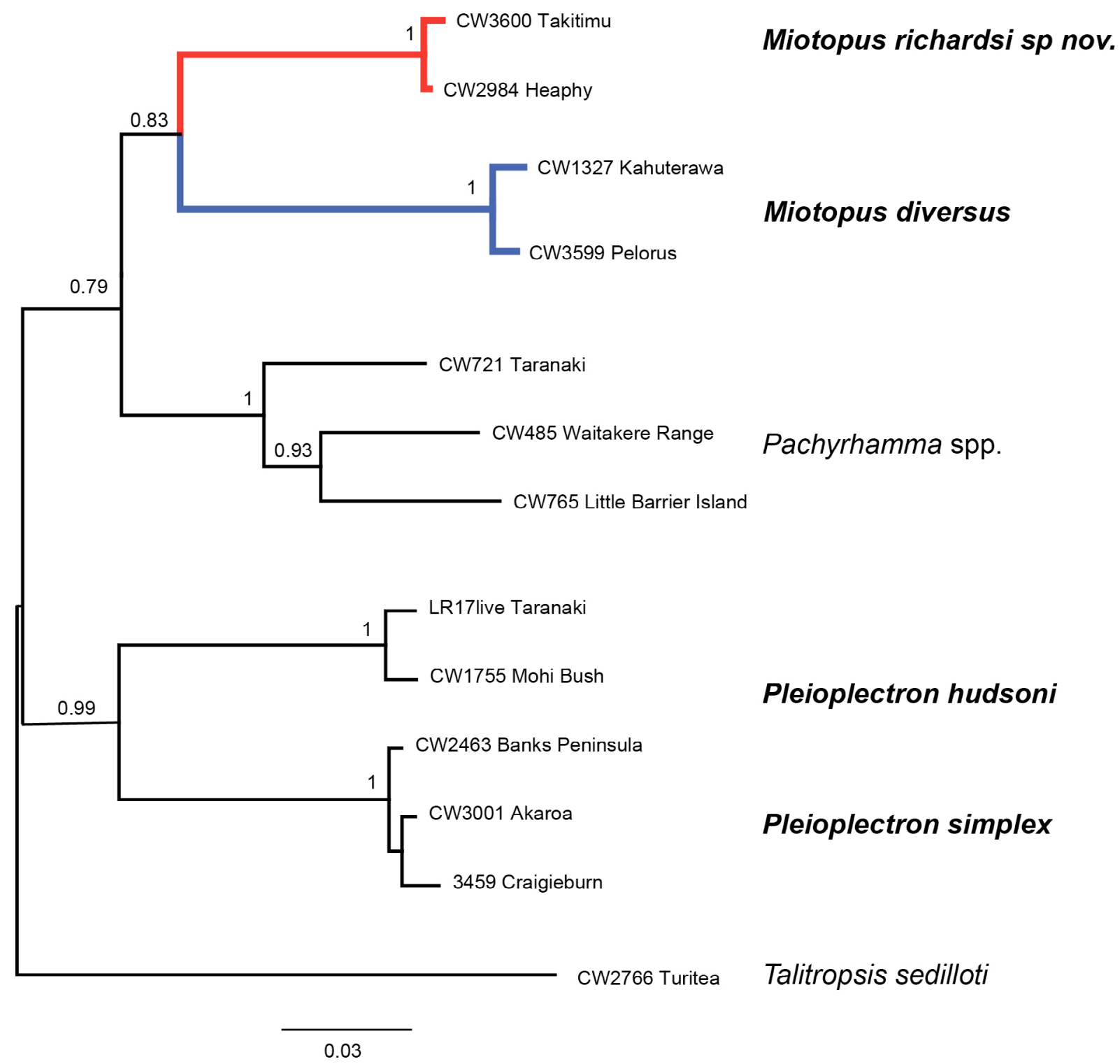

Fig. 3. Phylogenetic relationship of Pleioplectron Hutton, 1896 and Miotopus Hutton, 1898 inferred from Bayesian Inference of $1435 \mathrm{bp}$ mtDNA COI sequence alignment with four $4 \times 10^{6} \mathrm{MCMC}$ chains, sampling every $2 \times 10^{3}$ generations. 

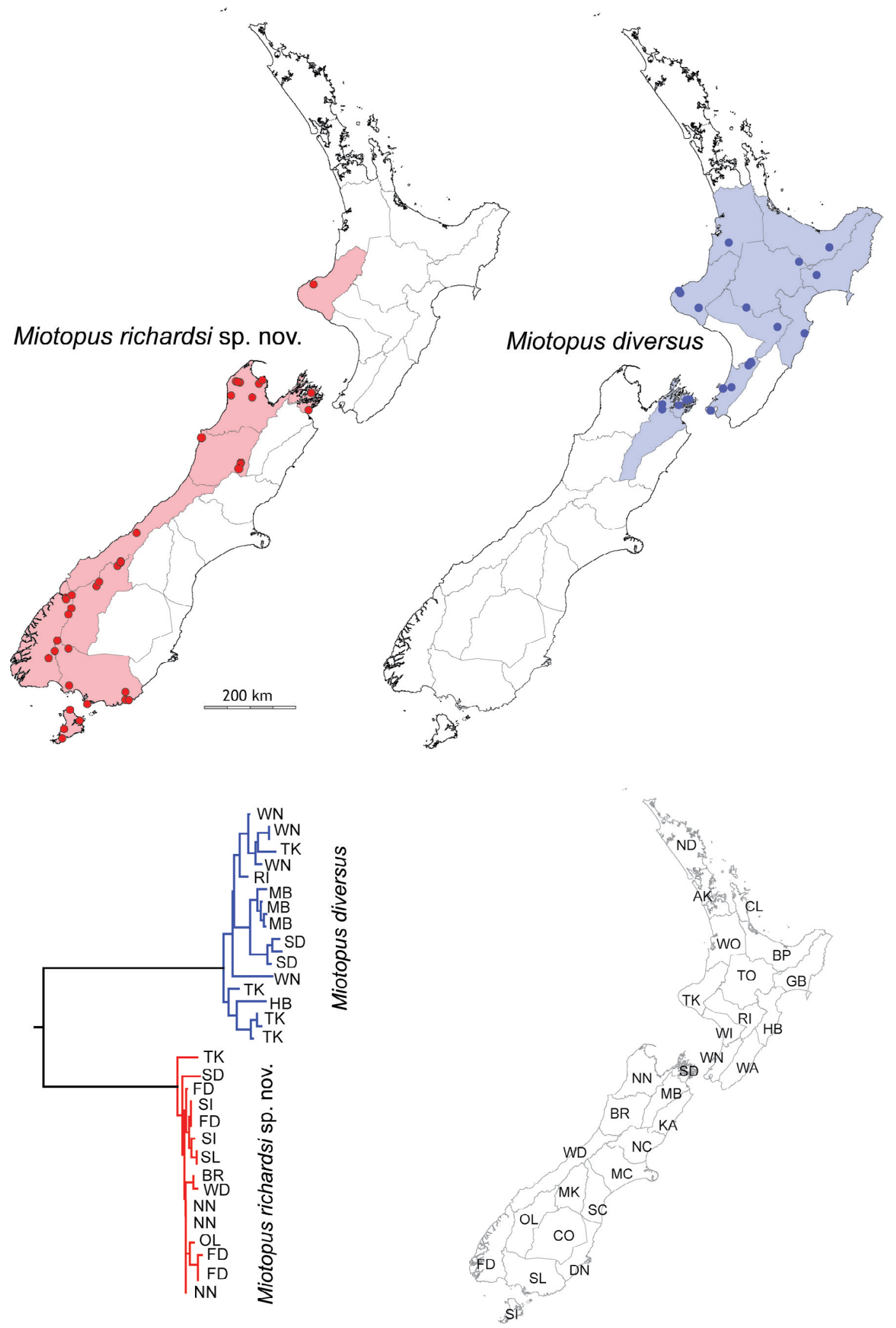

Fig. 4. Known distributions of Miotopus richardsi sp. nov. (red) and M. diversus (Hutton, 1896) (blue). Reciprocally monophyletic clusters of mtDNA sequences (835 bp COI, ML, HKY, with outgroup removed) of samples that encompass morphological and geographic diversity of the two taxa. Spot locations are indicated (circles) within the relevant New Zealand entomological (Crosby) regions. 
individuals collected on South Island. The other cluster consisted of specimens of a novel morphotype predominantly, but not exclusively from South Island (Fig. 4). We found mean genetic distances (HKY) between these putative Miotopus haplotype clusters of $0.110(\sim 11 \%)$, but 0.005 and 0.018 within them.

Given the morphological differences coupled with genetic evidence we agree with Hutton's (1898) decision to separate Pleioplectron and Miotopus and we redefine Miotopus here and describe a new southern species.

\author{
Order Orthoptera Latreille, 1793 \\ Superfamily Rhaphidophordoidea Walker, 1869 \\ Family Rhaphidophoridae Walker, 1869 \\ Subfamily Macropathinae Karny, 1930 \\ Tribe Macropathini Karny, 1930
}

Genus Miotopus Hutton, 1898

Medium size cave wētā (body length 11-17 mm) found in forests and caves, on three main islands of New Zealand. The genus consists of two species that are structurally quite distinct from one another, and share some morphological characteristics with Pleioplectron.

The head of Miotopus tends to be more elongated than in Pleioplectron, coloured mostly brown. Scapes of the antennae are sexually dimorphic, very broad in the males, thinner in the females (Fig. 5). Maxillary palps are long with moderately dense hair. The colour pattern of the upper body parts of the two species is similar, but darker in $P$. diversus, and surprisingly uniform across the whole distribution range with alternating reddish-brown and black patches. The light-coloured patches form a large inverted W-shape on the pronotum, and an X-shape stretching across the mesanotum, metanotum and first abdominal tergite (Fig. 6).

Legs are relatively long in both species, especially so in the newly described species. Fore and mid femora are armed at the apex with a prolateral and a retrolateral apical spine. Fore and mid tibia armed with two pairs of apical spines each, four pairs on the hind tibia. The number of linear spines on all tibiae varies within and between species (Table 1).

Male and female terminalia are species specific (Figs 7-8); cerci long and slender, especially in the males. The upper valves of the ovipositor are scabrous but not serrated (the irregularities being visible only at high magnification), while the lower valves have $7+$ shallow teeth near the apex (Fig. 8).

Miotopus diversus Hutton, 1898

Figs 4, 5A-B, 6A-B, 7A-B, 8A-C, 9, 10A-B

\title{
Diagnosis
}

A medium sized cave wêtā found in forested areas around the North Island, New Zealand, mainly in leaf litter on the forest floor, or in the roots of trees. Dark brown with visible dark and pale bands on the fore and mid legs, it could be most easily confused with the sympatric Pleioplectron hudsoni. However, adult Miotopus diversus are larger (see Table 1), usually appear darker in life, and have small spines on the dorsal surface of the mid tibiae, and are further distinguished from Pleioplectron by spine count and male terminalia. 


\section{Type material}

\section{Holotype}

NEW ZEALAND: + , from Upper Wanganui (as Pleioplectron diversum) in Canterbury Museum (Hutton 1897).

\section{Other material}

NEW ZEALAND: 1 from Makaretu, Hawkes Bay (as Miotopus diversus) (Hutton 1898).

\section{Material examined}

See Table 2.

\section{Description}

HEAD. Mostly brown with vertical pale stripes, covered in fine setae, palps light brown with fine setae, fastigium brown with pale spots on the sides, eyes black and ovoid, antennae long and dark brown. Male antennae are notably thick, densely clothed in setae and abruptly tapering to a thin thread at the end, whereas female antennae are narrow and almost uniformly thin from end to end, scape and peduncle pale (Fig. 5).

Thorax. Pronotum with anterior and posterior margins convex, and sides rounded with a slight outward 'lip', dark brown-red brown with occasional pale markings (Fig. 6A-B).

LEgs. Moderately long, hind femora shorter than tibiae, coxae and trochanters cream, femora and tibiae dark brown with cream bands. Fore femora compressed with one prolateral apical spine and one retrolateral apical spine present, short dark setae present. Fore tibiae with two prolateral and three or four retrolateral long, pale to transparent, linear spines positioned in the mid to distal portion of the tibiae. Fore tibiae with two superior apical spines (one prolateral and one retrolateral), pale with dark tip, almost hidden amongst the setae, two inferior apical spines (one prolateral and one retrolateral), inferior apical spines longer than superior spines, articulate, pale with dark tip, longer and thicker than the surrounding setae. Mid femora compressed with one long articulated prolateral apical spine, one long articulated retrolateral apical spine. Mid tibiae with three or four prolateral and two or three retrolateral long, linear spines positioned in the mid to distal portion of the tibiae, prolateral linear spines longer than the retrolateral spines. Mid tibiae with two superior apical spines (one prolateral and one retrolateral), pale with dark tip, two inferior apical spines (one prolateral and one retrolateral), inferior spines longer than superior spines, pale with dark tip. Hind femora with one retrolateral apical spine (sometimes very small), slightly pigmented. Hind tibiae longer than femora with small brown alternate spines along superior surface (Fig. 9). Hind tibiae with two superior subapical spines (one prolateral and one retrolateral), two superior apical spines (one prolateral and one retrolateral), spines twice as long as superior subapical spines, two inferior apical spines (one prolateral and one retrolateral) $2 / 3$ length of superior apical spines above, two inferior subapical spines. Tarsi with four segments, $1^{\text {st }}$ and $2^{\text {nd }}$ segment with a pair of spines on distal end, $1^{\text {st }}$ segment has eight small spines up from the end in alternate fashion; on the underside of the $1^{\text {st }}$ segment minute brown spinules run up the length of the segment either side of the tarsal pad. The $2^{\text {nd }}$ segment has three minute spines above, $4^{\text {th }}$ segment half the length of the $1^{\text {st }}$ segment.

AвDOMEn. Glossy, brown tones. Short setae covering both tergites and sternites; sternum light brown colour.

Terminalia male (Fig. 7A-B). Cerci long, round, brown in colour, clothed in setae, styli short, not extending beyond the end of the subgenital plate. Subgenital plate is a finger-like protrusion. 
Table 2 (continued on next pages). Material of Miotopus diversus (Hutton, 1896) examined. All specimens collected in New Zealand. Collector abbreviations: $\mathrm{DH}=$ Danilo Hegg; GB = Gareth Boyt; ML = Mike Lusk; MMR = Mary Morgan-Richards; RG $=$ Ron Goudswaard; ST $=$ Steve Trewick.

\begin{tabular}{|c|c|c|c|c|c|c|c|c|c|c|}
\hline $\begin{array}{c}\text { Entomological } \\
\text { Region }\end{array}$ & Material & Locality & Coordinates & $\begin{array}{c}\text { Elevation } \\
\text { m a.s.l. }\end{array}$ & $\begin{array}{l}\text { Micro- } \\
\text { habitat }\end{array}$ & $\begin{array}{c}\text { Collecting } \\
\text { method }\end{array}$ & Date & Collector & $\begin{array}{l}\text { Institution } \\
\text { code }\end{array}$ & $\begin{array}{l}\text { Specimen } \\
\text { code }\end{array}$ \\
\hline $\begin{array}{c}\text { TK } \\
\text { (Taranaki) }\end{array}$ & 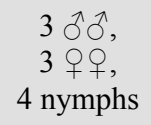 & $\begin{array}{c}\text { Egmont National Park, } \\
\text { Kaitake Range, } \\
\text { Lucy's Gully }\end{array}$ & $\begin{array}{l}-39.14931 \\
173.94167\end{array}$ & 200 & $\begin{array}{l}\text { Under log } \\
\text { in forest }\end{array}$ & Day search & 25 Sep. 2006 & ST \& MMR & MPN & CW459-CW465 \\
\hline $\begin{array}{c}\mathrm{TK} \\
\text { (Taranaki) }\end{array}$ & 1 우 & $\begin{array}{c}\text { Egmont National Park, } \\
\text { Oakura River }\end{array}$ & $\begin{array}{l}-39.19482 \\
173.99864\end{array}$ & 400 & & Night search & 1 Apr. 2007 & ST \& MMR & MPN & CW783 \\
\hline $\begin{array}{c}\text { TK } \\
\text { (Taranaki) }\end{array}$ & $\begin{array}{l}1 \hat{0} \\
3 \text { 우 }\end{array}$ & Lake Rotokare & $\begin{array}{l}-39.45409 \\
174.40923\end{array}$ & 200 & & Pitfall trap & & & LCR & $\begin{array}{l}\text { LR-L3-A } \\
\text { LR-W19-A, B } \\
\text { LR-L59-B }\end{array}$ \\
\hline $\begin{array}{c}\text { WN } \\
\text { (Wellington) }\end{array}$ & $10^{\lambda}$ & $\begin{array}{l}\text { Palmerston North, } \\
\text { Turitea Rd }\end{array}$ & $\begin{array}{l}-40.41566 \\
175.66390\end{array}$ & 100 & & & 21 Aug. 2004 & MMR & MPN & CW133 \\
\hline $\begin{array}{c}\text { WN } \\
\text { (Wellington) }\end{array}$ & $1+$ & $\begin{array}{c}\text { Palmerston North, } \\
\text { Turitea Rd }\end{array}$ & $\begin{array}{l}-40.41566 \\
175.66390\end{array}$ & 100 & & Night search & 15 Mar. 2005 & ST & MPN & CW336 \\
\hline $\begin{array}{c}\text { WN } \\
\text { (Wellington) }\end{array}$ & 10 & $\begin{array}{l}\text { Palmerston North, } \\
\text { Turitea Reserve }\end{array}$ & $\begin{array}{l}-40.43045 \\
175.67273\end{array}$ & 200 & $\begin{array}{l}\text { Near boat } \\
\text { shed }\end{array}$ & Night search & 15 Aug. 2010 & $\mathrm{ST}$ & MPN & CW1439 \\
\hline $\begin{array}{c}\text { WN } \\
\text { (Wellington) }\end{array}$ & $\begin{array}{c}4 \hat{\partial} \\
19\end{array}$ & $\begin{array}{l}\text { Palmerston North, } \\
\text { Turitea Reserve }\end{array}$ & $\begin{array}{l}-40.43045 \\
175.67273\end{array}$ & 200 & & Night search & 19 Nov. 2010 & $\mathrm{ST}$ & MPN & $\begin{array}{c}\text { CW 1452, } \\
\text { CW1459, } \\
\text { CW1467-CW1469 }\end{array}$ \\
\hline $\begin{array}{c}\text { WN } \\
\text { (Wellington) }\end{array}$ & $1 \delta^{\lambda}$ & $\begin{array}{l}\text { Palmerston North, } \\
\text { Turitea Reserve }\end{array}$ & $\begin{array}{l}-40.43045 \\
175.67273\end{array}$ & 200 & & Pitfall trap & & & & TD19(1)-b \\
\hline $\begin{array}{c}\text { WN } \\
\text { (Wellington) }\end{array}$ & 1 우 & $\begin{array}{l}\text { Palmerston North, } \\
\text { Kahuterawa Stream }\end{array}$ & $\begin{array}{l}-40.47068 \\
175.61204\end{array}$ & 150 & Stream bed & & 1 Jul. 2009 & & MPN & CW1327 \\
\hline $\begin{array}{c}\text { WN } \\
\text { (Wellington) }\end{array}$ & $1 \delta$ & $\begin{array}{l}\text { Wellington, } \\
\text { Karori Sanctuary }\end{array}$ & $\begin{array}{l}-41.302893 \\
174.703689\end{array}$ & 250 & & & 21 Jul. 2004 & $\mathrm{RG}$ & MPN & CW117 \\
\hline
\end{tabular}


Table 2 (continued).

\begin{tabular}{|c|c|c|c|c|c|c|c|c|c|c|}
\hline $\begin{array}{c}\text { Entomological } \\
\text { Region }\end{array}$ & Material & Locality & Coordinates & $\begin{array}{c}\text { Elevation } \\
\text { m a.s.l. }\end{array}$ & $\begin{array}{l}\text { Micro- } \\
\text { habitat }\end{array}$ & $\begin{array}{c}\text { Collecting } \\
\text { method }\end{array}$ & Date & Collector & $\begin{array}{l}\text { Institution } \\
\text { code }\end{array}$ & $\begin{array}{l}\text { Specimen } \\
\text { code }\end{array}$ \\
\hline $\begin{array}{c}\text { WN } \\
\text { (Wellington) }\end{array}$ & 10 & $\begin{array}{l}\text { Wellington, } \\
\text { Karori Sanctuary }\end{array}$ & $\begin{array}{l}-41.302893 \\
174.703689\end{array}$ & 250 & & & 11 Oct. 2004 & RG & MPN & CW182 \\
\hline $\begin{array}{c}\text { WN } \\
\text { (Wellington) }\end{array}$ & $1 \delta, 1$ 웅 & $\begin{array}{l}\text { Wellington, } \\
\text { Karori Sanctuary }\end{array}$ & $\begin{array}{l}-41.302893 \\
174.703689\end{array}$ & 250 & & & 22 Aug. 2006 & RG & MPN & CW475, CW476 \\
\hline $\begin{array}{c}\text { WN } \\
\text { (Wellington) }\end{array}$ & 1 우 & $\begin{array}{l}\text { Paraparaumu, } \\
\text { Nikau Reserve }\end{array}$ & $\begin{array}{l}-40.90624 \\
175.02093\end{array}$ & 20 & & & 20 Sep. 2004 & ST \& MMR & MPN & CW153 \\
\hline $\begin{array}{c}\text { WN } \\
\text { (Wellington) }\end{array}$ & $\begin{array}{c}1 \stackrel{+}{+} \\
\text { nymph }\end{array}$ & $\begin{array}{l}\text { Tararua Forest, } \\
\text { Otaki Forks }\end{array}$ & $\begin{array}{l}-40.88085 \\
175.21887\end{array}$ & 100 & $\begin{array}{c}\text { Under log } \\
\text { in forest }\end{array}$ & $\begin{array}{c}\text { Photograph } \\
\text { only }\end{array}$ & 4 Nov. 2017 & $\mathrm{DH}$ & iNaturalist & 8689928 \\
\hline $\begin{array}{c}\mathrm{RI} \\
\text { (Rangitikei) }\end{array}$ & 10 & $\begin{array}{c}\text { Central Ruahine, } \\
\text { Yeomans Track }\end{array}$ & $\begin{array}{l}-39.74383 \\
176.26003\end{array}$ & 400 & & & 10 Apr. 2011 & ML & MPN & CW1616 \\
\hline $\begin{array}{c}\text { HB } \\
\text { (Hawkes Bay) }\end{array}$ & 2 우우 & Mohi Bush & $\begin{array}{l}-39.85725 \\
176.90068\end{array}$ & 350 & & Night search & 8 Oct. 2012 & ST & MPN & CW1837, CW1841 \\
\hline $\begin{array}{c}\text { GB } \\
\text { (Gisborne) }\end{array}$ & 1 우 & Lake Waikaremoana & $\begin{array}{l}-38.797785, \\
177.120326\end{array}$ & 600 & $\begin{array}{l}\text { In hole in } \\
\text { tutu shrub }\end{array}$ & & 28 Mar. 2004 & MMR & MPN & CW75A \\
\hline $\begin{array}{c}\text { TO } \\
\text { (Taupo) }\end{array}$ & 1 우 & $\begin{array}{c}\text { Ruapehu, } \\
\text { Lake Rotokura }\end{array}$ & $\begin{array}{l}-39.42975 \\
175.52060\end{array}$ & 700 & & Night search & 11 Jul. 2006 & ST \& MMR & MPN & CW452 \\
\hline $\begin{array}{c}\text { TO } \\
\text { (Taupo) }\end{array}$ & 1 우 & Whirinaki Forest & $\begin{array}{l}-38.57248 \\
176.70391\end{array}$ & 400 & $\begin{array}{l}\text { On tree } \\
\text { trunk in } \\
\text { podocarp } \\
\text { forest }\end{array}$ & & 1 Jul. 2007 & GB & MPN & CW493 \\
\hline $\begin{array}{l}\text { BP (Bay } \\
\text { of Plenty) }\end{array}$ & $\begin{array}{c}2 \hat{\partial} \hat{O} \\
1 \hat{+}\end{array}$ & $\begin{array}{c}\text { Waioeka Gorge, } \\
\text { Manganuku }\end{array}$ & $\begin{array}{l}-38.290668 \\
177.385014\end{array}$ & 200 & $\begin{array}{c}\text { On } \\
\text { vegetation }\end{array}$ & Night search & 2 Jan. 2011 & ST & MPN & $\begin{array}{c}\text { CW1956, } \\
\text { CW1970, CW1971 }\end{array}$ \\
\hline $\begin{array}{c}\text { WO } \\
\text { (Waikato) }\end{array}$ & $10^{\pi}$ & $\begin{array}{c}\text { Waitomo, } \\
\text { Ruakuri Cave }\end{array}$ & $\begin{array}{l}-38.26652 \\
175.07721\end{array}$ & 100 & On tree & Night search & 4 Sep. 2012 & ST & MPN & CW1912 \\
\hline
\end{tabular}


Table 2 (continued).

\begin{tabular}{|c|c|c|c|c|c|c|c|c|c|c|}
\hline $\begin{array}{c}\text { Entomological } \\
\text { Region } \\
\end{array}$ & Material & Locality & Coordinates & $\begin{array}{c}\text { Elevation } \\
\text { m a.s.l. }\end{array}$ & $\begin{array}{l}\text { Micro- } \\
\text { habitat }\end{array}$ & $\begin{array}{c}\text { Collecting } \\
\text { method }\end{array}$ & Date & Collector & $\begin{array}{c}\text { Institution } \\
\text { code }\end{array}$ & $\begin{array}{l}\text { Specimen } \\
\text { code }\end{array}$ \\
\hline $\begin{array}{c}\text { MB } \\
\text { (Marlborough) }\end{array}$ & 1 ㅇ & $\begin{array}{l}\text { Pelorus Bridge, } \\
\text { Circle Loop Track }\end{array}$ & $\begin{array}{l}-41.29802 \\
173.57333\end{array}$ & 30 & & Night search & & ST \& MMR & MPN & CW533A \\
\hline $\begin{array}{c}\text { MB } \\
\text { (Marlborough) }\end{array}$ & $\begin{array}{l}2 \hat{\partial} \widehat{\partial} \\
1 \text { nymph }\end{array}$ & Pelorus Bridge & $\begin{array}{c}-41.29655 \\
173.5744\end{array}$ & 30 & $\begin{array}{l}\text { In roots of } \\
\text { fallen tree }\end{array}$ & $\begin{array}{l}\text { Night search } \\
+ \text { insect net }\end{array}$ & 6 Oct. 2017 & DH & MPN & $\begin{array}{l}\text { CW3598, } \\
\text { CW3599, } \\
\text { CW3790 }\end{array}$ \\
\hline $\begin{array}{c}\text { MB } \\
\text { (Marlborough) }\end{array}$ & 1 ㅇ & $\begin{array}{l}\text { Rai Valley, } \\
\text { Mt Richmond Estate }\end{array}$ & $\begin{array}{l}-41.20479 \\
173.57371\end{array}$ & 50 & $\begin{array}{l}\text { Pine / beech } \\
\text { forest }\end{array}$ & Day search & 15 Jan. 2011 & ST & MPN & CW1963 \\
\hline $\begin{array}{c}\text { SD } \\
\text { (Sounds) }\end{array}$ & $\begin{array}{l}2 \hat{\partial} \widehat{,} \\
1 \text { nymph }\end{array}$ & $\begin{array}{c}\text { Queen Charlotte } \\
\text { Sound, Resolution } \\
\text { Bay }\end{array}$ & $\begin{array}{l}-41.11118 \\
174.22488\end{array}$ & 0 & $\begin{array}{l}\text { Clay bank } \\
\text { on side of } \\
\text { track }\end{array}$ & $\begin{array}{l}\text { Night search } \\
+ \text { insect net }\end{array}$ & 1 Oct. 2017 & DH & MPN & $\begin{array}{c}\text { CW3601, } \\
\text { CW3602, CW3789 }\end{array}$ \\
\hline $\begin{array}{c}\text { SD } \\
\text { (Sounds) }\end{array}$ & $2+9+$ & $\begin{array}{l}\text { Queen Charlotte } \\
\text { Sound, Camp Bay }\end{array}$ & $\begin{array}{l}-41.12585 \\
174.14695\end{array}$ & 0 & $\begin{array}{l}\text { Clay bank } \\
\text { on side of } \\
\text { track }\end{array}$ & $\begin{array}{l}\text { Night search } \\
+ \text { insect net }\end{array}$ & 2 Oct. 2017 & DH & MPN & CW3596, CW3597 \\
\hline $\begin{array}{c}\text { SD } \\
\text { (Sounds) }\end{array}$ & 3 nymphs & $\begin{array}{l}\text { Queen Charlotte } \\
\text { Sound, Mistletoe Bay }\end{array}$ & $\begin{array}{l}-41.22236 \\
173.97254\end{array}$ & 40 & $\begin{array}{l}\text { Inside } \\
\text { hollow tree }\end{array}$ & $\begin{array}{l}\text { Casual find; } \\
\text { collected by } \\
\text { hand }\end{array}$ & 4 Oct. 2017 & DH & MPN & CW3805-CW3807 \\
\hline
\end{tabular}


Terminalia female (Fig. 8A-C). Subgenital plate with three points, the outer two slightly longer than the middle one. Apex rounded and blunt. Ovipositor reddish-brown with 7-8 teeth on the ventral edge near at the tip.

Miotopus richardsi sp. nov. urn:1sid:zoobank.org:act:BFDCEBC5-E058-4362-B5B4-42E3D9FAAD8F

Figs 4, 5C, 6C-D, 7C-F, 8D-F, 9, 10C-F

\section{Diagnosis}

A medium sized cave wētā found in forested areas of the South Island, New Zealand with a variegated colour pattern. Similar to Miotopus diversus based on apical spines with the exception of the presence on hind femora of both prolateral and retrolateral apical spines (n.b. this trait was formerly considered diagnostic of Pachyrhamma, see Cook et al. 2010). It is easily identified by the very long legs and the

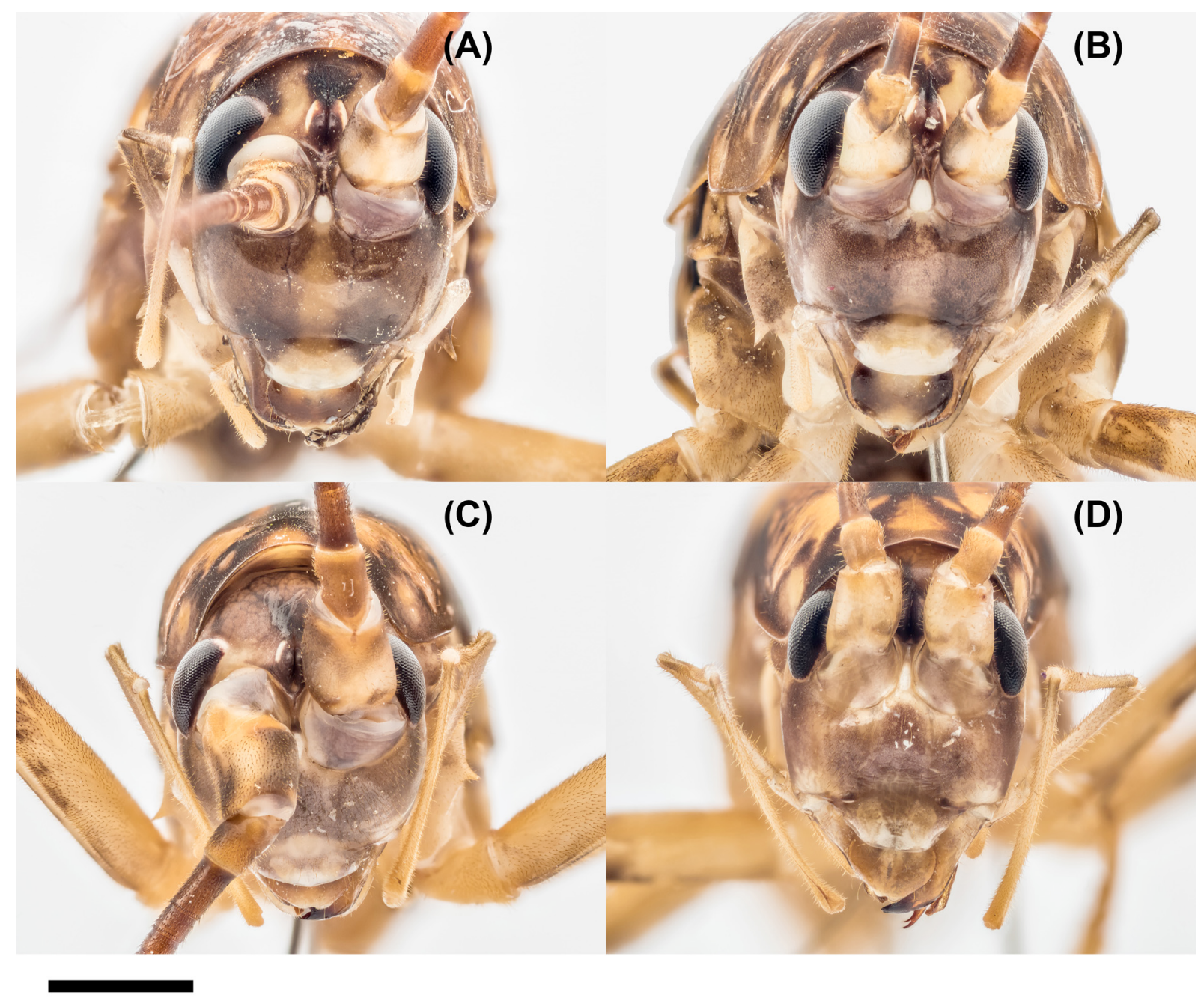

Fig. 5. Head of cave wētā in the genus Miotopus Hutton, 1898 showing sexual dimophism. A-B. Miotopus diversus (Hutton, 1896). A. Adult $\widehat{\jmath}$, Resolution Bay, Queen Charlotte Sound (MPN CW3459). B. Adult , Camp Bay, Queen Charlotte Sound (MPN CW3596). C-D. Miotopus richardsi sp. nov. Borland Road, Southland. C. Adult ổ (MPN CW3542). D. Adult $q$ (MPN CW3811). Scale bar $=2 \mathrm{~mm}$. 
presence of three pairs of prominent, socketed superior spines on the hind tibiae. Female with subgenital plate similar to $M$. diversus, but differs in male genital terminalia. Notably long ovipositor, as long as or longer than body length (Fig. 10C-F).

\section{Etymology}

Named for Aola Richards who studied New Zealand cave wētā and published many important systematic papers from 1954 until 1972.

\section{Type material}

\section{Holotype}

NEW ZEALAND: $\widehat{\jmath}$, adult, Fiordland (FD), Gulliver River, -44.704477, 167.97031, $60 \mathrm{~m}$ a.s.1., under large river boulders in native forest, by hand during night search, Jan. 2014, Tony Jewell leg. (MPN CW2619; NMNZ AI.035053).

\section{Paratype}

NEW ZEALAND: + , adult, Brunner (BR), Lewis Pass, Cannibal Gorge, -42.333962, 172.424113, $820 \mathrm{~m}$ a.s.1., on rotting tree stump in native forest, with insect net during night search, 24 Apr. 2017, Danilo Hegg leg. (MPN CW3429; NMNZ AI.035054).

\section{Additional material examined}

\section{See Table 3.}

\section{Description}

\section{Measurements. See Table 1.}

HEAD. Head slightly variegated colouring with a medium brown and golden light brown; covered in fine setae; palps are light golden brown and covered with fine setae; fastigium medium brown with minor golden light brown, with pale spots on the sides; eyes black; antennae long and medium brown with setae; scape and peduncle light golden brown (Fig. 5C-D).

Thorax. Pronotum anterior and posterior margins slightly convex; sides are rounded with a slight outward 'lip'. On the upper surface it bears the markings of a large inverted W, the side-arms fainter than the $\mathrm{V}$ in the middle (Fig. 6C-D).

Legs. Long. Hind femora shorter than tibiae. Coxae and trochanters cream in colour, femora and tibiae dark brown with cream bands. Fore femora compressed with one prolateral apical spine and one retrolateral apical spine, short dark setae present. Fore tibiae with two pairs of long, pale linear spines positioned in the mid to distal portion of the tibiae. Fore tibiae with two superior apical spines (one prolateral and one retrolateral), pale with dark tip and almost hidden amongst the setae, two inferior apical spines (one prolateral and one retrolateral), longer than superior spines, articulate, pale with dark tip, longer and thicker than the surrounding setae. Mid femora compressed with one long articulated prolateral apical spine, one long articulated retrolateral apical spine. Mid tibiae with two pairs of long, linear spines positioned in the mid to distal portion of the tibiae, prolateral spines longer than the retrolateral ones. Mid tibiae with two superior apical spines (one prolateral and one retrolateral), pale with dark tip, two inferior apical spines (one prolateral and one retrolateral), inferior spines longer than superior spines, pale with dark tip. Hind femora with one retrolateral and one prolateral apical spine, small, stout, slightly pigmented. Hind tibiae longer than femora with small brown alternate spines along length of the superior surface, three pairs are spurlike and three times the length of the other smaller spines (Fig. 9). Hind tibiae with two superior subapical spines (one prolateral and one retrolateral), two superior apical spines (one prolateral 


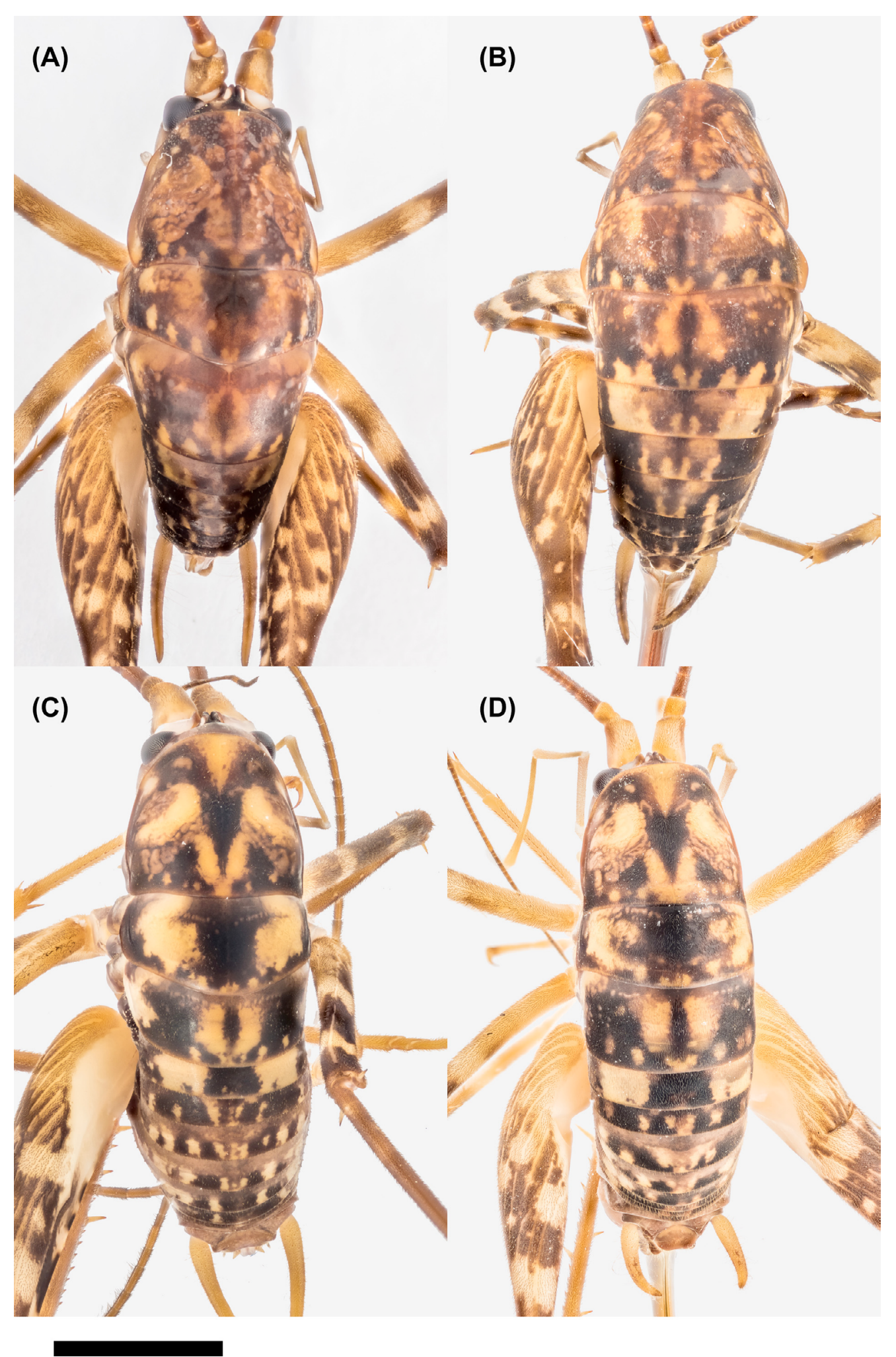

Fig. 6. Dorsal views of cave wētā in the genus Miotopus Hutton, 1898. A-B. Miotopus diversus (Hutton, 1896). A. Adult $\delta$, Resolution Bay, Queen Charlotte Sound (MPN CW3601). B. Adult $q$, Camp Bay, Queen Charlotte Sound (MPN CW3596). C-D. Miotopus richardsi sp. nov. C. Adult Oे, Lewis Pass (MPN CW3609). D. Adult +, Borland Road, Fiordland NP (MPN CW3811). Scale bar $=5 \mathrm{~mm}$. 
Table 3 (continued on next pages). Material of Miotopus richardsi sp. nov. examined. All specimens collected in New Zealand. ${ }^{\mathrm{H}}=$ Holotype, ${ }^{\mathrm{P}}=$ Paratype. Collector abbreviations: DC = Deborah Carden; DH = Danilo Hegg; MMR = Mary Morgan-Richards; ST = Steve Trewick; TJ = Tony Jewell.

\begin{tabular}{|c|c|c|c|c|c|c|c|c|c|c|}
\hline $\begin{array}{l}\text { Entomological } \\
\text { Region }\end{array}$ & Material & Locality & Coordinates & $\begin{array}{c}\text { Elevation } \\
\text { m a.s.1. }\end{array}$ & $\begin{array}{l}\text { Micro- } \\
\text { habitat }\end{array}$ & $\begin{array}{l}\text { Collecting } \\
\text { method }\end{array}$ & Date & Collector & $\begin{array}{l}\text { Institution } \\
\text { code }\end{array}$ & $\begin{array}{l}\text { Specimen } \\
\text { code }\end{array}$ \\
\hline $\begin{array}{c}\text { SI } \\
\text { (Stewart Island) }\end{array}$ & $2 \widehat{\partial} \sigma^{\lambda}$ & $\begin{array}{c}\text { Port Pegasus, } \\
\text { South Pegasus Hut }\end{array}$ & $\begin{array}{l}-47.199964, \\
167.638315\end{array}$ & 0 & Under tree arch & $\begin{array}{c}\text { Caught by } \\
\text { hand at night }\end{array}$ & 17 Jan. 2018 & DH & MPN & $\begin{array}{l}\text { CW3640, } \\
\text { CW3641 }\end{array}$ \\
\hline $\begin{array}{c}\text { SI } \\
\text { (Stewart Island) }\end{array}$ & 1 ㅇ & $\begin{array}{l}\text { Oban, } \\
\text { Horseshoe Point }\end{array}$ & $\begin{array}{l}-46.880401 \\
168.148586\end{array}$ & 0 & On tree & Night search & 20 Apr. 2017 & ST \& MMR & MPN & CW3481 \\
\hline $\begin{array}{c}\text { SI } \\
\text { (Stewart Island) }\end{array}$ & $1 \delta^{\lambda}$ & $\begin{array}{c}\text { Oban, } \\
\text { Raroa Walk }\end{array}$ & $\begin{array}{l}-46.900847 \\
168.120689\end{array}$ & 40 & On tree & Night search & 22 Apr. 2017 & ST \& MMR & MPN & CW3475 \\
\hline $\begin{array}{c}\text { SI } \\
\text { (Stewart Island) }\end{array}$ & $\begin{array}{c}1 \\
\text { nymph }\end{array}$ & $\begin{array}{l}\text { Yankee River } \\
\text { mouth }\end{array}$ & $\begin{array}{l}-46.694448, \\
167.891027\end{array}$ & 0 & $\begin{array}{l}\text { Coastal rocks } \\
\text { at bush edge }\end{array}$ & $\begin{array}{l}\text { Night search } \\
+ \text { insect net }\end{array}$ & 15 Apr. 2018 & DH & MPN & CW3809 \\
\hline $\begin{array}{c}\text { SI } \\
\text { (Stewart Island) }\end{array}$ & $1 \sigma^{\lambda}$ & Doughboy Bay & $\begin{array}{l}-47.033764 \\
167.708637\end{array}$ & 0 & Sea cave & $\begin{array}{l}\text { Night search } \\
+ \text { insect net }\end{array}$ & 21 Apr. 2018 & DH & MPN & CW3808 \\
\hline $\begin{array}{c}\text { SL } \\
\text { (Southland) }\end{array}$ & $1 \widehat{\sigma}$ & $\begin{array}{l}\text { Catlins Forest, } \\
\text { McLean Falls }\end{array}$ & $\begin{array}{l}-46.57132 \\
169.34773\end{array}$ & 50 & $\begin{array}{l}\text { Rocks on side of } \\
\text { walking track }\end{array}$ & $\begin{array}{l}\text { Night search } \\
+ \text { insect net }\end{array}$ & 22 Oct. 2017 & DH & MPN & CW3604 \\
\hline $\begin{array}{c}\text { SL } \\
\text { (Southland) }\end{array}$ & $\begin{array}{c}1 \delta^{\lambda} \\
\text { nymph }\end{array}$ & $\begin{array}{l}\text { Catlins Forest, } \\
\text { Lake Wilkie }\end{array}$ & $\begin{array}{l}-46.57957 \\
169.43991\end{array}$ & 10 & $\begin{array}{l}\text { On tree trunk in } \\
\text { podocarp forest }\end{array}$ & $\begin{array}{l}\text { Night search } \\
+ \text { insect net }\end{array}$ & 22 Oct. 2017 & DH & MPN & CW3603 \\
\hline $\begin{array}{c}\text { SL } \\
\text { (Southland) }\end{array}$ & $\begin{array}{c}1 \overbrace{}^{\lambda} \\
\text { nymph }\end{array}$ & $\begin{array}{l}\text { Catlins Forest, } \\
\text { McLennan Hut }\end{array}$ & $\begin{array}{l}-46.43216 \\
169.36144\end{array}$ & 200 & Inside rotting log & $\begin{array}{l}\text { Photograph } \\
\text { only }\end{array}$ & 12 Dec. 2015 & DH & iNaturalist & 2626726 \\
\hline $\begin{array}{c}\text { SL } \\
\text { (Southland) }\end{array}$ & 1 우 & $\begin{array}{c}\text { Takitimu Forest, } \\
\text { Lower Princhester } \\
\text { Hut }\end{array}$ & $\begin{array}{l}-45.59397 \\
167.95193\end{array}$ & 470 & On tree trunk & $\begin{array}{l}\text { Night search } \\
+ \text { insect net }\end{array}$ & 21 Oct. 2017 & DH & MPN & CW3600 \\
\hline $\begin{array}{c}\text { SL } \\
\text { (Southland) }\end{array}$ & $1 q$ & $\begin{array}{l}\text { Longwood Range, } \\
\text { Cascade Creek }\end{array}$ & $\begin{array}{l}-46.260298 \\
167.907572\end{array}$ & 500 & $\begin{array}{l}\text { Under wooden } \\
\text { bridge }\end{array}$ & Night search & 2013 & $\mathrm{TJ}$ & MPN & CW2567 \\
\hline $\begin{array}{c}\text { SL } \\
\text { (Southland) }\end{array}$ & $3 \partial^{\top} \sigma^{-1}$ & Bluff Hill & $\begin{array}{l}-46.620853 \\
168.348742\end{array}$ & 50 & $\begin{array}{l}\text { Rata tree trunk } \\
\text { and shrubs }\end{array}$ & Night search & Aug. 2013 & $\mathrm{TJ}$ & MPN & $\begin{array}{l}\text { CW2556, } \\
\text { CW2557, } \\
\text { CW2564 }\end{array}$ \\
\hline $\begin{array}{c}\text { FD } \\
\text { (Fiordland) }\end{array}$ & $1 \widehat{\sigma}$ & $\begin{array}{c}\text { Kepler Track, } \\
\text { Dock Bay }\end{array}$ & $\begin{array}{l}-45.42701 \\
167.609053\end{array}$ & 200 & Beech logs & Day search & 29 Dec. 2009 & $\mathrm{ST}$ & MPN & CW972 \\
\hline $\begin{array}{c}\text { FD } \\
\text { (Fiordland) }\end{array}$ & 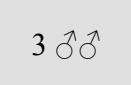 & Cleddau River & $\begin{array}{l}-44.677947, \\
167.963227\end{array}$ & 20 & In road culvert & Night search & Jan. 2014 & $\mathrm{TJ}$ & MPN & $\begin{array}{l}\text { CW2616- } \\
\text { CW2618 }\end{array}$ \\
\hline
\end{tabular}


Table 3 (continued).

\begin{tabular}{|c|c|c|c|c|c|c|c|c|c|c|}
\hline $\begin{array}{l}\text { Entomological } \\
\text { Region }\end{array}$ & Material & Locality & Coordinates & $\begin{array}{c}\text { Elevation } \\
\text { m a.s.l. }\end{array}$ & $\begin{array}{l}\text { Micro- } \\
\text { habitat }\end{array}$ & $\begin{array}{c}\text { Collecting } \\
\text { method }\end{array}$ & Date & Collector & $\begin{array}{l}\text { Institution } \\
\text { code }\end{array}$ & $\begin{array}{c}\text { Specimen } \\
\text { code }\end{array}$ \\
\hline $\begin{array}{c}\text { FD } \\
\text { (Fiordland) }\end{array}$ & $1 ठ 1 \%$ & Gulliver River & $\begin{array}{c}-44.704477 \\
167.97031\end{array}$ & 60 & $\begin{array}{l}\text { Under dry boulder } \\
\text { overhang in forest }\end{array}$ & Night search & Jan. 2014 & $\mathrm{TJ}$ & MPN & $\begin{array}{c}\mathrm{CW} 2619^{\mathrm{H}} \\
\mathrm{CW} 2620\end{array}$ \\
\hline $\begin{array}{c}\text { FD } \\
\text { (Fiordland) }\end{array}$ & $1 \widehat{\delta}, 1$ + & Lake Rakatu & $\begin{array}{l}-45.62037 \\
167.59631\end{array}$ & 190 & $\begin{array}{l}\text { On tree trunk in } \\
\text { native forest }\end{array}$ & $\begin{array}{c}\text { Caught by } \\
\text { hand at night }\end{array}$ & 10 Sep. 2016 & $\mathrm{DH}$ & MPN & $\begin{array}{l}\text { CW3810, } \\
\text { CW3523 }\end{array}$ \\
\hline $\begin{array}{c}\text { FD } \\
\text { (Fiordland) }\end{array}$ & $1 ठ 1$ ㅇ & Borland Rd & $\begin{array}{l}-45.74031 \\
167.42700\end{array}$ & 700 & $\begin{array}{l}\text { Dry rock overhang } \\
\text { in road cutting }\end{array}$ & $\begin{array}{l}\text { Night search } \\
+ \text { insect net }\end{array}$ & 3 Mar. 2017 & $\mathrm{DH}$ & MPN & $\begin{array}{l}\text { CW3542, } \\
\text { CW3811 }\end{array}$ \\
\hline $\begin{array}{c}\text { FD } \\
\text { (Fiordland) }\end{array}$ & $1 \delta^{\lambda}$ & $\begin{array}{l}\text { Eglinton Valley, } \\
\text { Knobs Flat }\end{array}$ & $\begin{array}{l}-44.97774 \\
168.00520\end{array}$ & 350 & $\begin{array}{c}\text { In cavity at base } \\
\text { of tree trunk }\end{array}$ & $\begin{array}{l}\text { Photograph } \\
\text { only }\end{array}$ & 13 Jan. 2009 & $\mathrm{DH}$ & iNaturalist & 3101320 \\
\hline $\begin{array}{c}\text { FD } \\
\text { (Fiordland) }\end{array}$ & 1 우 & $\begin{array}{l}\text { Eglinton Valley, } \\
\text { Lake Gunn }\end{array}$ & $\begin{array}{l}-44.87218 \\
168.08828\end{array}$ & 480 & In shrubs & $\begin{array}{l}\text { Night search } \\
+ \text { insect net }\end{array}$ & 11 Mar. 2017 & $\mathrm{DH}$ & MPN & CW3543 \\
\hline $\begin{array}{c}\text { OL } \\
\text { (Otago Lakes) }\end{array}$ & $1 \hat{\delta}, 1$ + & $\begin{array}{l}\text { West Matukituki } \\
\text { River, } \\
\text { Raspberry Flat }\end{array}$ & $\begin{array}{l}-44.50037 \\
168.75445\end{array}$ & 450 & $\begin{array}{c}\text { Under rock } \\
\text { overhang in bluffs }\end{array}$ & $\begin{array}{c}\text { Caught by } \\
\text { hand at night }\end{array}$ & 21 Feb. 2016 & $\mathrm{DH}$ & MPN & $\begin{array}{l}\text { CW2848, } \\
\text { CW2849 }\end{array}$ \\
\hline $\begin{array}{c}\text { OL } \\
\text { (Otago Lakes) }\end{array}$ & $1 \sigma^{\lambda}$ & $\begin{array}{l}\text { East Matukituki } \\
\text { River }\end{array}$ & $\begin{array}{l}-44.42167 \\
168.82668\end{array}$ & 400 & On beech tree trunk & $\begin{array}{l}\text { Photograph } \\
\text { only }\end{array}$ & 22 Feb. 2016 & $\mathrm{DH}$ & iNaturalist & 8021131 \\
\hline $\begin{array}{c}\text { OL } \\
\text { (Otago Lakes) }\end{array}$ & $\begin{array}{c}1 \delta \\
\text { nymph }\end{array}$ & $\begin{array}{l}\text { Makarora River, } \\
\text { Cameron Flat }\end{array}$ & $\begin{array}{l}-44.15611 \\
169.30472\end{array}$ & 370 & On beech tree trunk & $\begin{array}{l}\text { Night search } \\
+ \text { insect net }\end{array}$ & 1 Sep. 2017 & $\mathrm{DH}$ & MPN & CW3701 \\
\hline $\begin{array}{c}\text { OL } \\
\text { (Otago Lakes) }\end{array}$ & $\begin{array}{l}3 \hat{\jmath} \\
2 \text { 우우 }\end{array}$ & $\begin{array}{c}\text { Hollyford Track } \\
\text { upstream of } \\
\text { Hidden Falls }\end{array}$ & $\begin{array}{l}-44.63550 \\
168.11785\end{array}$ & 50 & $\begin{array}{l}\text { Under logs or rocks } \\
\text { on side of track }\end{array}$ & $\begin{array}{l}\text { Night search } \\
+ \text { insect net }\end{array}$ & 12 Nov. 2016 & DH & MPN & $\begin{array}{l}\text { CW3138- } \\
\text { CW3140, } \\
\text { CW3605, } \\
\text { CW3606 }\end{array}$ \\
\hline $\begin{array}{c}\text { WD } \\
\text { (Westland) }\end{array}$ & $1 \delta^{\lambda}$ & Copland Track & $\begin{array}{l}-43.57632 \\
169.81347\end{array}$ & 50 & & & 14 Jan. 2004 & ST & MPN & CW52 \\
\hline $\begin{array}{c}\text { BR } \\
\text { (Brunner) }\end{array}$ & 1 운 & $\begin{array}{c}\text { Charleston, } \\
\text { Mitchell's } \\
\text { Gully Gold Mine }\end{array}$ & $\begin{array}{c}-41.89194, \\
171.4677783\end{array}$ & 50 & $\begin{array}{l}\text { In mining } \\
\text { tunnel }\end{array}$ & $\begin{aligned} & \text { Torch } \\
+ & \text { insect net }\end{aligned}$ & 30 Jan. 2018 & $\mathrm{DH}$ & MPN & CW3763 \\
\hline $\begin{array}{c}\text { BR } \\
\text { (Brunner) }\end{array}$ & $1 \delta^{\lambda}$ & Lewis Pass & $\begin{array}{l}-42.37139 \\
172.40372\end{array}$ & 800 & $\begin{array}{l}\text { In leaf litter } \\
\text { on forest floor }\end{array}$ & $\begin{array}{l}\text { Night search } \\
+ \text { insect net }\end{array}$ & 25 Apr. 2017 & $\mathrm{DH}$ & MPN & CW3609 \\
\hline $\begin{array}{c}\text { BR } \\
\text { (Brunner) }\end{array}$ & 2 우우 & $\begin{array}{l}\text { Lewis Pass, } \\
\text { Cannibal Gorge }\end{array}$ & $\begin{array}{l}-42.34845 \\
172.41506\end{array}$ & 820 & $\begin{array}{l}\text { On rotting tree } \\
\text { stump }\end{array}$ & $\begin{array}{l}\text { Night search } \\
+ \text { insect net }\end{array}$ & 25 Apr. 2017 & $\mathrm{DH}$ & MPN & $\begin{array}{l}\text { CW3429, } \\
\text { CW3610 }\end{array}$ \\
\hline
\end{tabular}


Table 3 (continued).

\begin{tabular}{|c|c|c|c|c|c|c|c|c|c|c|}
\hline $\begin{array}{c}\text { Entomological } \\
\text { Region }\end{array}$ & Material & Locality & Coordinates & $\begin{array}{c}\text { Elevation } \\
\text { m a.s.l. }\end{array}$ & $\begin{array}{l}\text { Micro- } \\
\text { habitat }\end{array}$ & $\begin{array}{l}\text { Collecting } \\
\text { method }\end{array}$ & Date & Collector & $\begin{array}{l}\text { Institution } \\
\text { code }\end{array}$ & $\begin{array}{l}\text { Specimen } \\
\text { code }\end{array}$ \\
\hline $\begin{array}{c}\mathrm{BR} \\
\text { (Brunner) }\end{array}$ & 10 & $\begin{array}{l}\text { Lewis Pass, } \\
\text { Nina Valley }\end{array}$ & $\begin{array}{l}-42.459738 \\
172.364717\end{array}$ & 600 & $\begin{array}{l}\text { Under large rock } \\
\text { in forest }\end{array}$ & $\begin{array}{l}\text { Night search } \\
+ \text { insect net }\end{array}$ & 26 Jan. 2018 & DH & MPN & CW3762 \\
\hline $\begin{array}{c}\mathrm{NN} \\
\text { (Nelson) }\end{array}$ & 10 & $\begin{array}{c}\text { Oparara, } \\
\text { Box Canyon Cave }\end{array}$ & $\begin{array}{l}-41.13481 \\
172.19008\end{array}$ & 250 & In cave & & Feb. 2005 & $\mathrm{DC}$ & MPN & CW345 \\
\hline $\begin{array}{c}\mathrm{NN} \\
\text { (Nelson) }\end{array}$ & 1 ㅇ & $\begin{array}{l}\text { Takaka, } \\
\text { Sky Farm }\end{array}$ & $\begin{array}{l}-40.92435 \\
172.85541\end{array}$ & 600 & Beech tree trunk & Night search & 29 Jan. 2006 & ST \& MMR & MPN & CW302 \\
\hline $\begin{array}{c}\mathrm{NN} \\
\text { (Nelson) }\end{array}$ & $\begin{array}{c}1 \\
\text { nymph }\end{array}$ & $\begin{array}{c}\text { Takaka Hill, } \\
\text { Summit Walkway }\end{array}$ & $\begin{array}{c}-41.032057 \\
172.86519\end{array}$ & 800 & Hole in stick & Day search & 20 Jan. 2018 & ST & MPN & CW3630 \\
\hline $\begin{array}{c}\mathrm{NN} \\
\text { (Nelson) }\end{array}$ & $10^{\pi}$ & $\begin{array}{c}\text { Abel Tasman NP, } \\
\text { Awapoto Hut }\end{array}$ & $\begin{array}{l}-40.86314 \\
172.93910\end{array}$ & 650 & On shrub branchlets & $\begin{array}{l}\text { Night search } \\
+ \text { insect net }\end{array}$ & 11 Oct. 2017 & DH & MPN & CW3812 \\
\hline $\begin{array}{c}\mathrm{NN} \\
\text { (Nelson) }\end{array}$ & $\begin{array}{c}101 \text { } \\
7 \\
\text { nymphs }\end{array}$ & $\begin{array}{l}\text { Heaphy Track, } \\
\text { Gouland Downs } \\
\text { Caves }\end{array}$ & $\begin{array}{l}-40.89180 \\
172.35237\end{array}$ & 620 & Under cave ceiling & $\begin{array}{l}\text { Torch }+ \\
\text { insect net }\end{array}$ & 21 Apr. 2016 & DH & MPN & $\begin{array}{l}\text { CW3007, } \\
\text { CW3008, } \\
\text { CW3019- } \\
\text { CW3025 }\end{array}$ \\
\hline $\begin{array}{c}\mathrm{NN} \\
\text { (Nelson) }\end{array}$ & 1 우 & $\begin{array}{l}\text { Heaphy Track, } \\
\text { Three Pointer }\end{array}$ & $\begin{array}{l}-40.88281 \\
172.30926\end{array}$ & 800 & On tree trunk & $\begin{array}{l}\text { Night search } \\
+ \text { insect net }\end{array}$ & 22 Apr. 2016 & DH & MPN & CW2984 \\
\hline $\begin{array}{c}\text { NN } \\
\text { (Nelson) }\end{array}$ & 1 우 & $\begin{array}{l}\text { Heaphy Track, } \\
\text { Perry Saddle }\end{array}$ & $\begin{array}{l}-40.90010 \\
172.40756\end{array}$ & 900 & $\begin{array}{l}\text { Under overhang in } \\
\text { bank on side of track }\end{array}$ & $\begin{array}{l}\text { Night search } \\
+ \text { insect net }\end{array}$ & 19 Apr. 2016 & DH & MPN & CW2989 \\
\hline $\begin{array}{c}\mathrm{NN} \\
\text { (Nelson) }\end{array}$ & 1011 운 & $\begin{array}{l}\text { Heaphy Track, } \\
\text { Perry Saddle }\end{array}$ & $\begin{array}{l}-40.90010 \\
172.40756\end{array}$ & 900 & $\begin{array}{l}\text { Under overhang in } \\
\text { bank on side of track }\end{array}$ & $\begin{array}{l}\text { Night search } \\
+ \text { insect net }\end{array}$ & 24 Dec. 2016 & DH & MPN & $\begin{array}{l}\text { CW3607, } \\
\text { CW3608 }\end{array}$ \\
\hline $\begin{array}{c}\mathrm{NN} \\
\text { (Nelson) }\end{array}$ & 1 ㅇ & $\begin{array}{l}\text { Mt Arthur, Lower } \\
\text { Gridiron Shelter }\end{array}$ & $\begin{array}{c}-41.1704017 \\
172.69670\end{array}$ & 800 & $\begin{array}{l}\text { Under large boulder } \\
\text { in forest }\end{array}$ & $\begin{array}{l}\text { Night search } \\
+ \text { insect net }\end{array}$ & 9 Feb. 2018 & DH & MPN & CW3764 \\
\hline $\begin{array}{c}\text { SD } \\
\text { (Sounds) }\end{array}$ & 1 우 & Rarangi Beach & $\begin{array}{l}-41.39430 \\
174.04489\end{array}$ & 0 & In cave & & 11 Dec. 2007 & MMR & MPN & CW2430 \\
\hline $\begin{array}{c}\text { SD } \\
\text { (Sounds) }\end{array}$ & $\begin{array}{c}1 \stackrel{\circ}{+} \\
\text { nymph }\end{array}$ & Mt Stokes & $\begin{array}{c}-41.088055 \\
174.105\end{array}$ & 1160 & $\begin{array}{l}\text { In leaf litter on } \\
\text { forest floor }\end{array}$ & $\begin{array}{l}\text { Night search } \\
+ \text { insect net }\end{array}$ & 29 Jan. 2018 & DH & MPN & CW3772 \\
\hline $\begin{array}{c}\text { TK } \\
\text { (Taranaki) }\end{array}$ & 1 우 & $\begin{array}{c}\text { New Plymouth, } \\
\text { Lake Mangamahoe }\end{array}$ & $\begin{array}{l}-39.12904 \\
174.12570\end{array}$ & 180 & Hollow ponga & Day search & 11 Nov. 2006 & ST \& MMR & MPN & CW1403 \\
\hline
\end{tabular}




\section{Miotopus diversus}
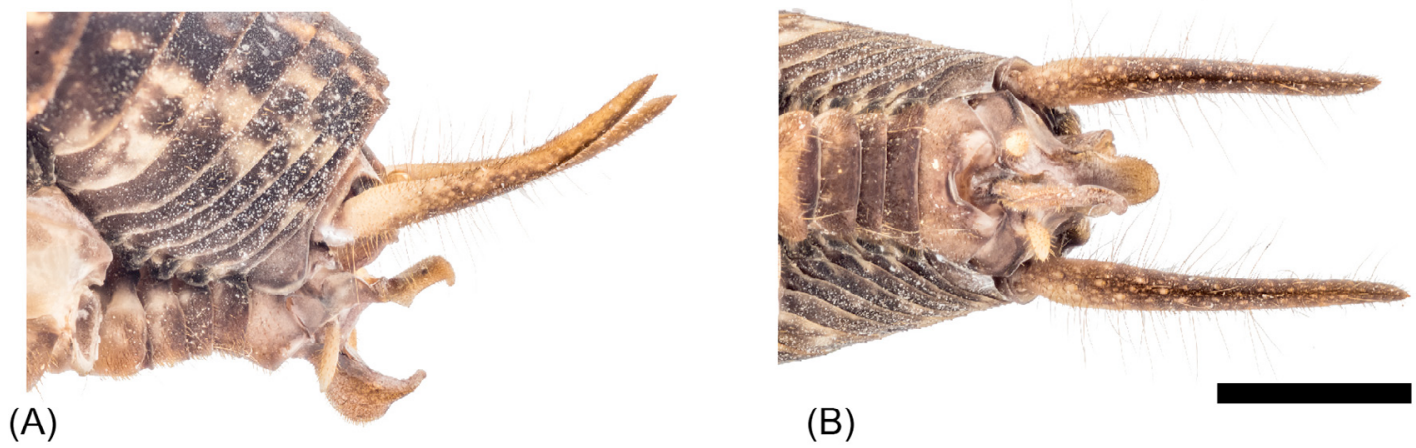

(B)

Miotopus richardsi sp. nov.

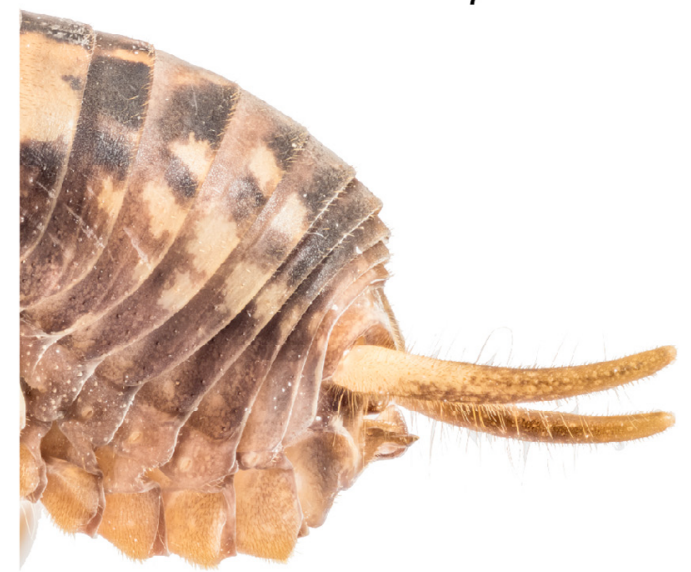

(C)

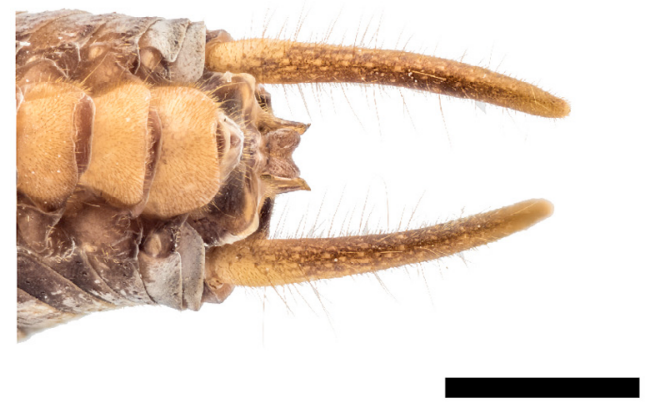

(D)
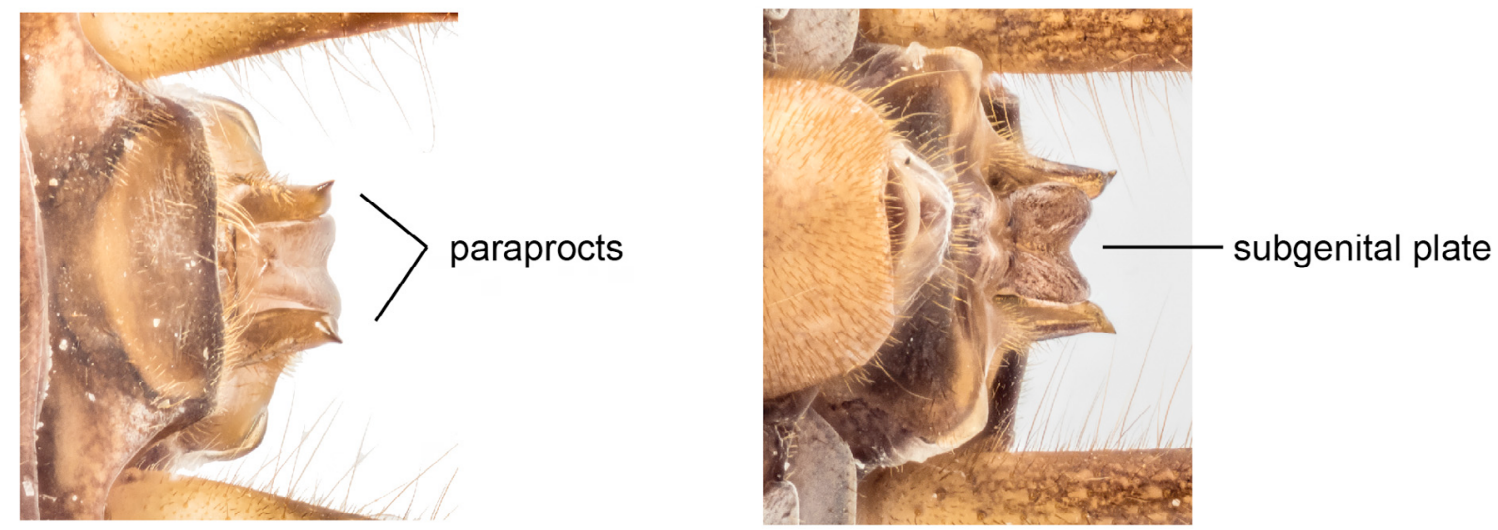

$(\mathrm{E})$

(F)

Fig. 7. Male terminalia of Miotopus Hutton, 1898 cave wētā. A-B. Miotopus diversus (Hutton, 1896) (MPN CW3601). A. Lateral. B. Ventral. C-F. Miotopus richardsi sp. nov. (MPN CW3542). C. Lateral. D. Ventral. E. Close dorsal. F. Ventral views of named structures. Scale bars $=2 \mathrm{~mm}$. 
and one retrolateral), twice as long as superior subapical spines, two inferior apical spines (one prolateral and one retrolateral) $2 / 3$ the length of superior apical spines above, two inferior subapical spines. Hind tarsi with 4 segments, $1^{\text {st }}$ and $2^{\text {nd }}$ segment with a pair of spines on distal end. $1^{\text {st }}$ segment has 4-11 small dorsal, alternate spines, and minute brown spinules along the underside of either side of the tarsal pad. The $2^{\text {nd }}$ segment occasionally has one or two small spines above, $4^{\text {th }}$ segment half the length of the $1^{\text {st }}$ segment.

AвDOMEn. Shiny, brown coloured. Short setae covering both tergites and sternites; sternum pale brown colour.

Terminalia male. Cerci long, round, brown in colour, clothed in setae. Styli reduced, not extending beyond the end of the subgenital plate. Subgenital plate short and bulbous, usually with median groove on ventral surface. Paraprocts adjacent to subgenital plate each bearing a prominent, sharp spine (Fig. 7C-D).

Terminalia Female. Subgenital plate simple. Appears as either truncated or sinuous with middle portion of posterior margin slightly indented. Ovipositor very long, as long as or longer than the body length, reddish-brown with 18-30 small teeth on ventral edge near the tip (Fig. 8D-F).

\section{Miotopus diversus}

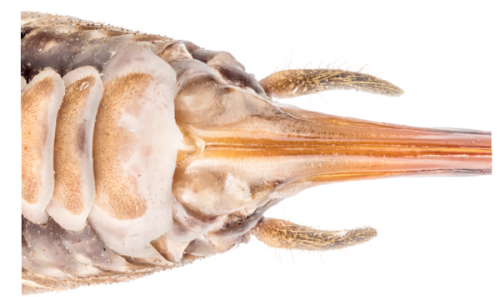

(A)

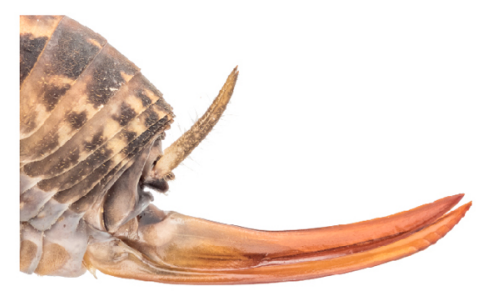

(B)

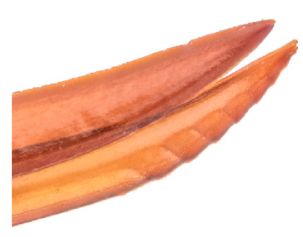

(C)

\section{Miotopus richardsi sp. nov.}

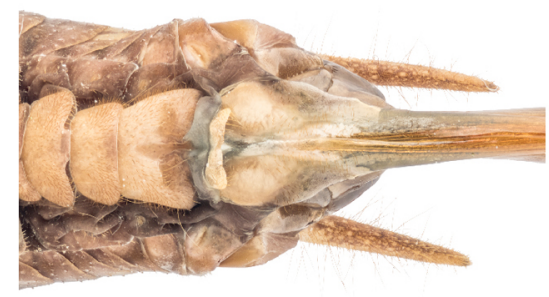

(D)

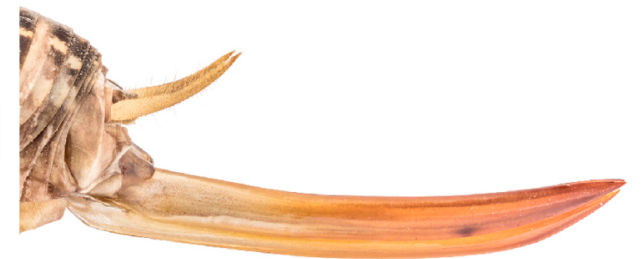

$(\mathrm{E})$

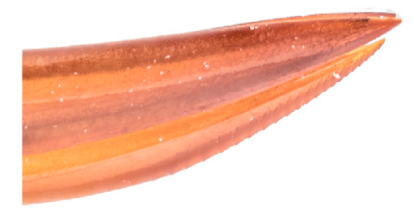

$(\mathrm{F})$

Fig. 8. Female terminalia of Miotopus Hutton, 1898 cave wētā. A-C. Miotopus diversus (Hutton, 1896) (MPN CW3596). A. Ventral. B-C. Lateral views. D-F. Miotopus richardsi sp. nov. (MPN CW3543). D. Ventral. E-F. lateral views. Scale bars $=3 \mathrm{~mm}$. 


\section{Discussion}

Miotopus richardsi sp. nov. is found in forest habitat and in caves; it is both common and widespread on South Island, from the Catlins Forest across Southland to Fiordland, and the length of the West Coast and of the Southern Alps to Kahurangi and Abel Tasman National Parks (Fig. 4). It is also found on Stewart Island, and in the Taranaki Region on North Island.

Adults have long legs, longer than in all other South Island forest species, except for Macropathus Walker (1869). Just like Macropathus, it is almost always found during the day suspended upside down, most commonly in cavities at the base of tree trunks, under boulders, branches and logs, under rocky overhangs and in caves. It is very light sensitive and will quickly get away with long leaps if disturbed. In open forest, individuals are rarely found close together. However, dense colonies occur under rocky overhangs and in caves. Under a roof in the bluffs at Raspberry Flat, West Matukituki, fifty to a hundred specimens were found in close proximity to each other, crossing legs and antennae. In the Gouland Downs caves in Kahurangi National Park, the population of Miotopus richardsi sp. nov. reaches hundreds if not thousands of individuals, often in direct physical contact with each other. Here they mix with Pachyrhamma edwardsi (Scudder 1869) and, in lower densities, Macropathus filifer (Walker 1869). Pachyrhamma delli (Richards 1954) is also found in the same caves, but occupies small fissures and limestone pockets, and does not mix with the other species of cave wētā.

The body colouring pattern is surprisingly uniform across the whole species' distribution range (Fig. 10C, E-F). Only cave dwelling specimens are generally paler (Fig. 10D). Nymphs look very much like the adults in general colouring, with the 3 pairs of prominent spines on the hind tibiae already noticeable in specimens just a few mm long. This makes the species very easy to recognize. Females have a strong, long and relatively straight ovipositor, as long as, or longer than the body length. They use it to penetrate soft wood in rotting logs, where they lay their eggs (Fig. 10E). Small nymphs of Miotopus richardsi sp. nov. have been found right in the core of large rotting logs, suggesting that this is an environment in which they commonly spend the early developmental stages.

Obtaining information about diet depends on sporadic observations, but in M. richardsi sp. nov., as in other cave wētā the diet includes fungal and vegetable matter. We documented M. richardsi sp. nov.

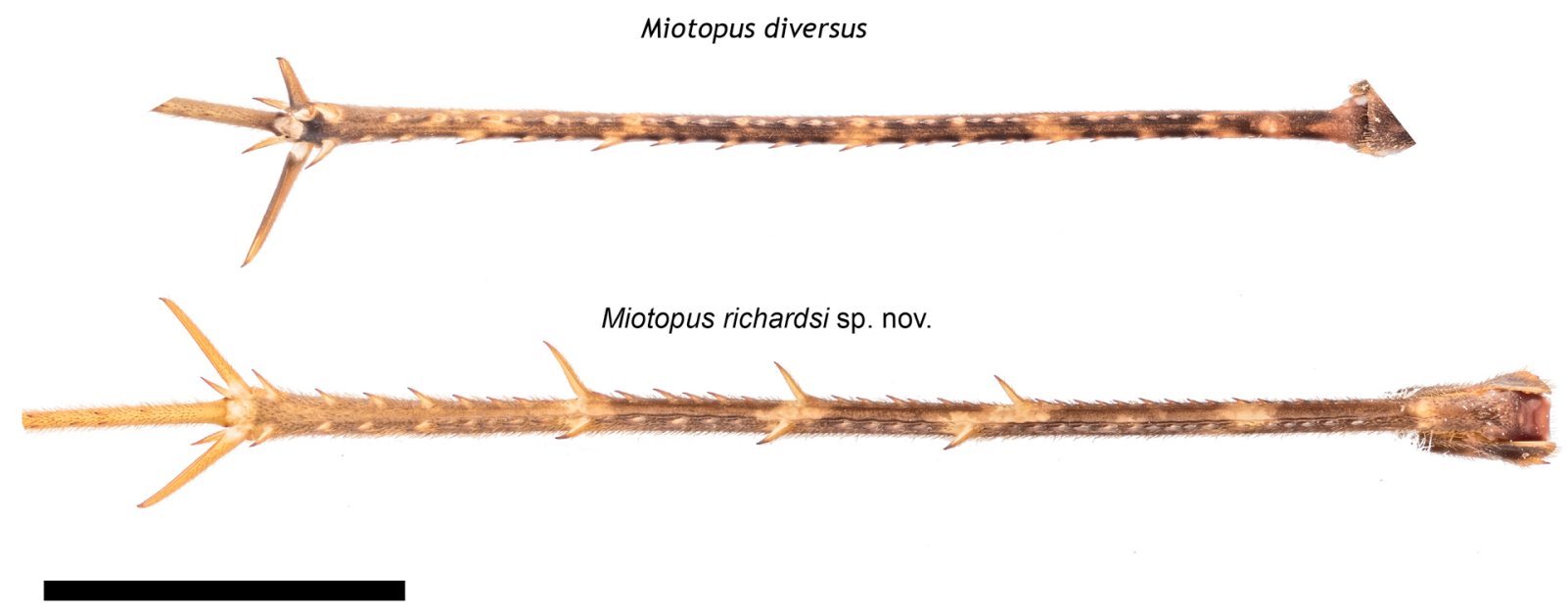

Fig. 9. Left hind tibia of cave wētā in the genus Miotopus Hutton, 1898, dorsal view. Miotopus diversus (Hutton, 1896) (MPN CW3601) (top) and Miotopus richardsi sp. nov. (MPN CW3543) (bottom). Scale bar $=5 \mathrm{~mm}$. 
feeding on lichen and leafy liverworts (Plagiochila spp.) on tree trunks, and on fungal fruiting bodies on the forest floor. It was also observed feeding on a beech strawberry fungus Cyttaria gunnii Berk., a meal it was sharing with a darkling beetle Zeadelium intricatum (Broun, 1880). We have also documented an adult Miotopus apparently obtaining moisture or slime from the head of a leaf-veined slug (Pseudaneitea spp.), while straddling it with its long legs (Fig. 10F), and have seen this behaviour in several other species of cave wētā.
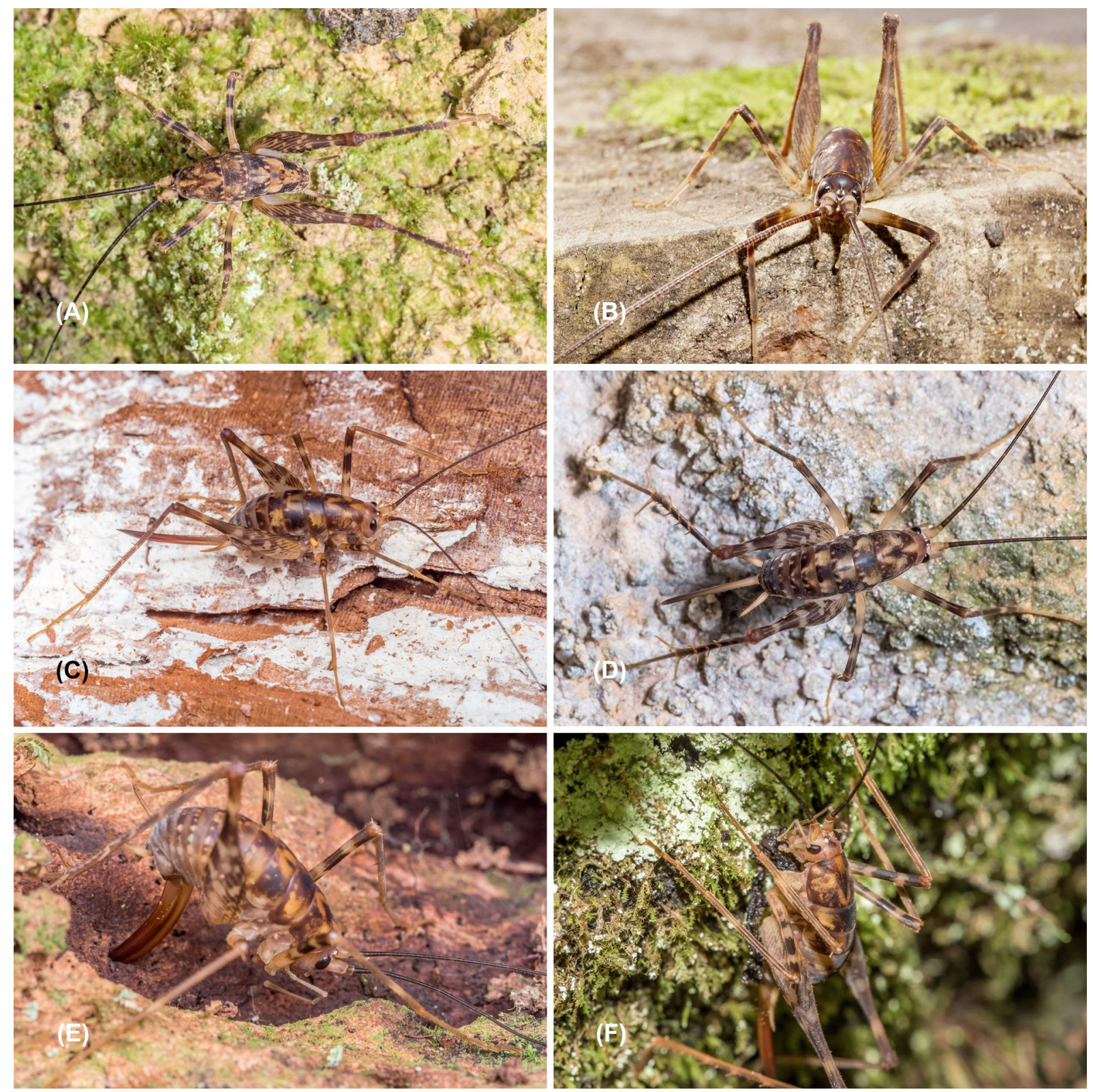

Fig. 10. Living Miotopus Hutton, 1898 in their natural environment. A-B. Miotopus diversus (Hutton, 1896), adult Ŝ. A. Resolution Bay, Queen Charlotte Sound. B. Turitea Reserve, Palmerston North. C-F. Miotopus richardsi sp. nov. C. Adult ${ }_{\text {, }}$ Brewster Hut Track, Haast Pass. D. Female nymph, Gouland Downs Caves, Kahurangi NP. E. + , laying eggs in rotting wood, Brewster Hut Track, Haast Pass. F. O, 'licking' the slime off a native leaf-veined slug (Pseudaneitea spp.), Raspberry Flat, Matukituki River West Branch. 


\section{Acknowledgements}

Thanks to the New Zealand Department of Conservation for collecting permits and support of this biodiversity research. We thank the many enthusiasts who contributed specimens including Gareth Boyt, Deborah Carden, Ron Goudswaard, Tony Jewell, Mike Lusk, Amy McKenzie, Briar Taylor-Smith, Stephen Thorpe and Edward Trewick.

\section{References}

Allegrucci G., Trewick S.A., Fortunato A., Carchini G. \& Sbordoni V. 2010. Cave crickets and cave weta (Orthoptera) from the southern end of the world: a molecular phylogeny test of biogeographical hypotheses. Journal of Orthoptera Research 19: 121-130. https://doi.org/10.1665/034.019.0118

Cook L., Trewick S.A., Morgan-Richards M. \& Johns P. 2010. Status of the New Zealand cave weta (Rhaphidophoridae) genera Pachyrhamma, Gymnoplectron and Turbottoplectron. Invertebrate Systematics 24: 131-138. https://doi.org/10.1071/IS09047

Fitness J.L., Morgan-Richards M., Ball O.J.-P., Godfrey A.J.R. \& Trewick S.A. 2015. Improved resolution of cave weta diversity (Orthoptera: Rhaphidophoridae): ecological implications for Te Paki, Far North, New Zealand. New Zealand Journal of Zoology 42: 1-16. https://doi.org/10.1080/03014223.2014.983939

Folmer O., Black M., Hoeh W. \& Lutz R.V.R. 1994. DNA primers for the amplification of mitochondrial cytochrome c oxidase subunit I from diverse metaxoan invertebrates. Molecular Marine Biology and Biotechnology 3: 294-299.

Hutton F.W. 1897. The Stenopelmatidae of New Zealand. Transactions of the New Zealand Institute 29: $208-242$.

Hutton F.W. 1898. Supplement to the Stenopelmatidae of New Zealand. Transactions and Proceedings of the New Zealand Institute 31: 40-43.

Kearse M., Moir R., Wilson A., Stones-Havas S., Cheung M., Sturrock S., Buxton S., Cooper A., Markowitz S., Duran C., Thierer T., Ashton B., Meintjes P. \& Drummond A. 2012. Geneious Basic: an integrated and extendable desktop software platform for the organization and analysis of sequence data. Bioinformatics 28: 1647-1649. https://doi.org/10.1093/bioinformatics/bts199

Richards A.M. 1954. The systematics and ecology of the genus Macropathus Walker, 1869 (Orthoptera, Rhaphidophoridae). Transactions of the Royal Society of New Zealand 82: 739-762.

Richards A.M. 1959. Revision of the Rhaphidophoridae (Orthoptera) of New Zealand - Part V. The genus Pleioplectron Hutton 1897. Transactions of the Royal Society of New Zealand 87: 319-327.

Scudder S.H. 1869. A new cave insect from New Zealand. Proceedings of the Boston Society of Natural History 12: 408-409.

Simon C., Frati F., Beckenbach A., Crespi B.J., Liu H. \& Flook P. 1994. Evolution, weighting, and phylogenetic utility of mitochondrial gene sequences and a compilation of conserved polymerase chain reaction primers. Annals of the Entomological Society of America 87: 651-701. https://doi.org/10.1093/aesa/87.6.651

Trewick S.A. \& Morgan-Richards M. 2005. After the deluge: mitochondrial DNA indicates Miocene radiation and Pliocene adaptation of tree and giant weta (Orthoptera: Anostostomatidae). Journal of Biogeography 32: 295-309. https://doi.org/10.1111/j.1365-2699.2004.01179.x

Walker F. 1869. Catalogue of the specimens of Dermaptera Saltatoria and Supplement to the Blattariae in the collection of the British Museum Volume 1. Trustees of the British Museum, London.

https://doi.org/10.5962/bhl.title.28190 
Manuscript received: 19 June 2018

Manuscript accepted: 7 August 2018

Published on: 18 October 2018

Topic editor: Gavin Broad

Desk editor: Pepe Fernández

Printed versions of all papers are also deposited in the libraries of the institutes that are members of the EJT consortium: Muséum national d'Histoire naturelle, Paris, France; Botanic Garden Meise, Belgium; Royal Museum for Central Africa, Tervuren, Belgium; Natural History Museum, London, United Kingdom; Royal Belgian Institute of Natural Sciences, Brussels, Belgium; Natural History Museum of Denmark, Copenhagen, Denmark; Naturalis Biodiversity Center, Leiden, the Netherlands; Museo Nacional de Ciencias Naturales-CSIC, Madrid, Spain; Real Jardín Botánico de Madrid CSIC, Spain; Zoological Research Museum Alexander Koenig, Bonn, Germany. 